\title{
A novel congestion control mechanism for multicast real-time connections
}

\author{
Jau-Hsiung Huang, Chun-Chuan Yang*, Nai-Cheng Fang \\ Communications and Multimedia Laboratory Department of Computer Science and Information Engineering National Taiwan University, \\ Taipei, Taiwan R.O.C.
}

Received 8 August 1997; received in revised form 24 September 1998; accepted 24 September 1998

\begin{abstract}
Time framing strategies such as Stop-and-Go (S\&G) [Golestani S.J., Congestion-free transmission of real-time traffic of real-time traffic in packet networks, IEEE INFOCOM, 1990, pp.527-536; Golestani S.J., A framing strategy for congestion management, IEEE JSAC, 9(7), 1991, pp.1064-1077; Golestani S.J., Congestion-free communication in high-speed packet networks, IEEE Trans. on Communications, 39(12), 1991, pp.1802-1812; Golestani S.J., A stop-and-go queueing framework for congestion management, ACM SIGCOMM, 1992, pp.818] and Continuous Framing (CF) [Jau-Hsiung Huang, Biau-Jwo Tsaur, Continuous framing mechanism for congestion control in broadband networks, Computer Communications, 18(10), 1995, pp.718-724] are designed to support the end-to-end delay bound and jitter bound for unicast connections. Considering the features of real-time multicast connections, a new time framing mechanism named Multicast Continuous Framing (MCF) is proposed in this article. S\&G and CF allow only one time frame length per connection, but MCF allows changing of the time frame length of a connection at intermediate nodes so that the statistical multiplexing gain within a connection is increased, i.e., the bandwidth requirement can be reduced. We also present the multicast connection setup scheme for MCF in the article. Simulation results show that MCF has a much better performance than that of $\mathrm{CF}$, and the tighter jitter bound a connection requests, the more performance improvement MCF can obtain. (C) 1999 Elsevier Science B.V. All rights reserved.
\end{abstract}

Keywords: Real-time; Stop- and -Go; MCF

\section{Introduction}

The problem of congestion control has been the subject of extensive research for computer networks. Traditionally, acknowledgment-based control, such as window-based flow control, makes use of the information fed by the downstream or the destination node to regulate the input traffic. In broadband networks, the propagation delay, when measured in terms of the service time of a packet, is much longer than that in narrowband networks; consequently, the acknowledgement-based control mechanism is not suitable.

In addition, the window-based flow control and firstcome-first-serve policy can neither satisfy some performance requirements, such as bounded end-to-end delay and loss free transmissions, nor provide firewalls among connections. Thus, some rate-based congestion control mechanisms [1-5] have been proposed. These control mechanisms include Fair Queueing (FQ) [6], Hierarchical Round Robin (HRR) [7], Real-Time Virtual Clock (RealTime VC) [8, 9], Delay-Earliest-Due-Date (Delay-EDD) [10], and Jitter-Earliest-Due-Date (Jitter-EDD) [11], Rate

* Corresponding author. Tel.: + 88649910960 ext. 4131; fax: + 886 4 2116689; e-mail: ccyang@csie.ncnu.edu.tw
Controlled Static Prioriy (RCSP) [12], Stop-and-Go (S and G) [13-16], Continuous Framing (CF) [17], and Generalized Processor Sharing [19, 20].

Among these control mechanisms, S\&G [13-16] guarantees a bounded end-to-end delay and loss free transmission for real-time applications. Bandwidth reservation for each connection is determined by the admission control based on peak rate requirement. $S \& G$ adopts a time framing strategy to separate time into frames. Under the framing architecture, $S \& G$ requires that packets arriving in the $k$ th frame should be sent out in the $(k+1)$ th frame as shown in Fig. 1, in which the time frame length is assumed to be $T$. In this way, packets will not suffer more than $T$ queuing time at each intermediate node and therefore a bounded delay can be provided along the path. In S\&G, it is desirable to incorporate multiple frame sizes according to different delay requirements. However, the frame size of longer ones must be integer multiples of that of the smaller ones.

As the arriving frame and the departing frame of a switch node are not always synchronized as in Fig. 1, S\&G introduces an extra delay, which is called the synchronization delay, for each packet to smooth the skew between arriving frame and the departing frame. Hence, the synchronization delay incurs a larger end-to-end delay. 


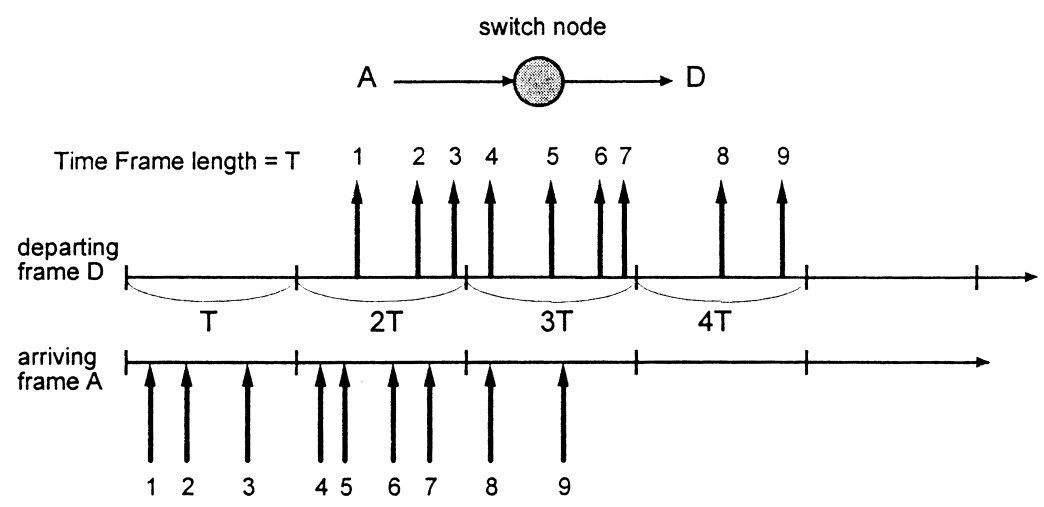

Fig. 1. The concept of Stop-and-Go.

The CF [17] scheme was proposed to eliminate the synchronization delay incurred in S\&Gsuch that the endto-end delay bound is reduced from $2 \mathrm{HT}$ to HT, where $H$ is the hop count of the connection. In order to remove the synchronization delay, the time framing structures of the incoming and the outgoing links should be perfectly synchronized. That is, for a connection $j$ which passes through an intermediate node $i$ with incoming link $L_{\text {in }}$ and outgoing link $L_{\text {out }}$, node $i$ should start a time frame on $L_{\text {out }}$ for connection $j$ as soon as a time frame finishes on $L_{\text {in }}$. To achieve this, CF requires the source node to send packets uniformly within a frame as shown in Fig. 2, and adds an end flag in the last packet within a frame to identify the end of the time frame, as packets 3 and 7 in the figure. Thus, as long as the switch node sees a packet with the end flag, it knows that the incoming frame has been finished and a new frame should be started immediately on the outgoing link. The packets are then transmitted uniformly over the new frame.

With this mechanism, the time frames of different connections on a link do not need to begin or end at the same time. As the framing structures on $L_{\text {in }}$ and $\mathrm{L}_{\text {out }}$ are perfectly synchronized, there will be no synchronization delay at each node. Under such a mechanism, a packet will suffer only a delay of $T$ on each intermediate node,

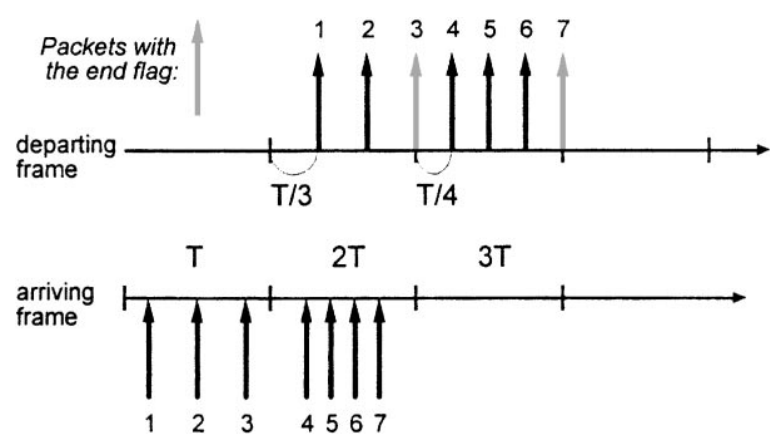

Fig. 2. CF: sending packets uniformly within a frame.
Hence the end-to-end delay can be bounded by HT. Other than that if the end-to-end delay bound is reduced by half, CF can improve the link utilization by $40 \%$ for many cases as shown in [17].

Both $S \& G$ and $C F$ were designed for unicast connections. When applying them to multicast connections, we found that the network resources could be easily wasted. A typical example of the routing tree for a multicast connection is shown in Fig. 3. It is reasonable to assume that the end-toend delay requirements for all destinations of the multicast connection are the same. We denote the delay requirement as $D$. Using CF, the time frame length $T$ of the connection is determined by the path from the source to the farthest destination, as the path S-R2 in Fig. 3. Hence, $T$ should not be larger than $D / 5$ in this case where 5 is the link count between $\mathrm{S}$ and R2. However, the bounded delay on the path from $\mathrm{S}$ to $\mathrm{R} 1$ will equal $D^{*} 3 / 5$, which is smaller than what is required. If we enlarge the frame lengths of links $L_{1}$ and $L_{2}$ to $2 \mathrm{~T}$, the delay requirement from $\mathrm{S}$ to $\mathrm{R} 1$ is still met while the efficiency of links $L_{1}$ and $L_{2}$ can also be improved. This is because the larger the time frame, the more statistical multiplexing gain we can get from a connection; i.e. less bandwidth is required for the connection.

$\mathrm{CF}$ provides only one time frame length for a connection along the path, and it does not allow the switch node to change the frame length once the length is decided. Therefore, $\mathrm{CF}$ needs to be modified to improve the efficiency of network utilization for multicast connections as explained above. A modification of CF, named as Multicast Continuous Framing $(M C F)$ scheme, is proposed in the article to allow the length of the time frame to be changed in switch nodes.

The article is organized as described next. The concept of $\mathrm{MCF}$ is given in section 2, and the admission control, endto-end delay, and jitters of MCF are also presented in the section. The connection setup scheme for multicast connections is presented in section 3 . In section 4 , we present several simulation results of MCF for performance evaluation. Lastly, section 5 concludes this article. 


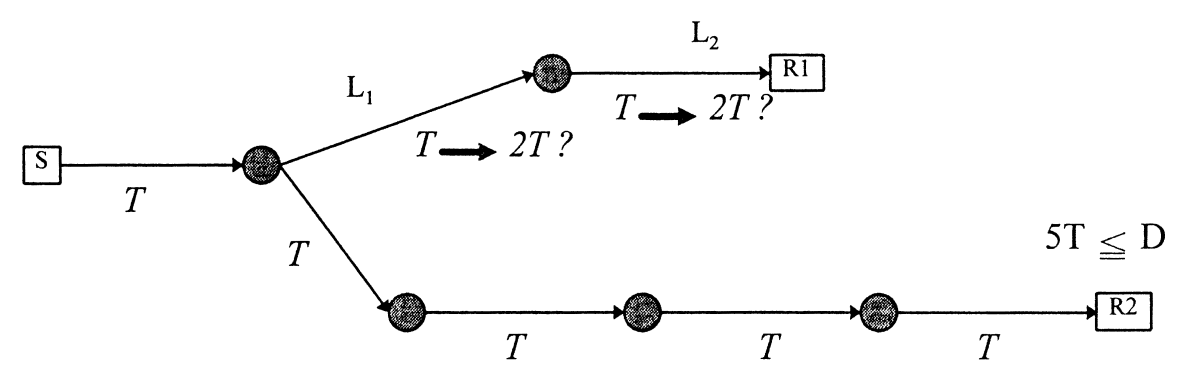

Fig. 3. An example of multicast routing tree.

\section{Multicast continuous framing}

\subsection{General concept of $M C F$}

MCF adopts the same framing concept as CF does, i.e. sending packets uniformly in a time frame and using end flags to indicate the ends of time frames. The objective of MCF is to allow changes of the time frame length along the path of a connection. In MCF, each connection selects its own set of time frame lengths. The frame length of longer ones in the set is assumed to be integer multiples of that of the smaller ones. That is, if a connection selects $G$ time frame lengths, $T_{1}>T_{2}>\ldots>T_{G}$, they satisfy the following relationship: $T_{i}=K_{i} * T_{i+1}, K_{i} \in N, i=1 \sim \mathrm{G}-1$.

The basic idea of MCF is to record the information of all time frame lengths in the header of each packet. More specifically, MCF identifies each kind of time frame used in a connection by the end flag of each time frame in the packet header. For example, a packet with the end flags of time frames $T_{i}$ and $T_{j}$ implies the packet is the end packet of both $T_{i}$ and $T_{j}$. The operations in MCF are divided into two parts: at the source node and at the intermediate nodes. The source node performs the actions of recording all time frame information in the packets, and the intermediate nodes perform the actions of changing the time frame if required according to the information recorded in the packets. Next we present the control actions of the source and the intermediate nodes.

\subsubsection{MCF control at the source node}

As there is only one frame length allowed along the path of a connection in CF, packets only need a one-bit field to indicate if the packet carries an end flag. MCF allows a set of time frames used for a connection, so the packet in MCF must have one end bit for each kind of time frame. The flow control field of a MCF packet is shown in Fig. 4. There are G end flag bits followed by a group number in the flow control field in which $G$ is the number of frame sizes used in the connection. The group number is used in changing the time frame at the intermediate nodes, and the physical interpretation of the group number is explained next.
We assume time frames $T_{1}, T_{2}, \ldots, \mathrm{T}_{\mathrm{G}}$ are used for a connection and $T_{i}=K_{i} * T_{i+1}$ as mentioned in section 2.1. $T_{\max }$ and $T_{\min }$ are assumed to be the maximum time frame and the minimum time frame respectively; i.e., $T_{\max }=T_{1}$ and $T_{\min }=T_{\mathrm{G}} . T_{\text {out }}$ denotes the selected output time frame of the source node.

In order to set the end flags of all time frames for input packets, the arriving packets must be buffered $T_{\max }$ time at the source node. The source node adds end flags in the end packets of different time frames $\mathrm{T}_{1}, \mathrm{~T}_{2}, \ldots, \mathrm{T}_{\mathrm{G}}$, and records the values of the group number in each packet, in which we define the last packet arrived in a time frame as the end packet of that frame. The group number of a packet in the buffered $T_{\max }$ time is the sequence number of the $T_{\min }$ frame which the packet is in. For example, suppose $T_{\max }=4 T_{\min }$ which means there are $4 T_{\min }$ frames within the buffered $T_{\max }$ time at the source node, the group number of a packet arrived in the first $T_{\min }$ frame has a value of 1 and the group number of a packet arrived in the second $T_{\min }$ frame has a value of 2, and the group number of a packet arrived in the last $T_{\min }$ frame has a value of 4 . The assignment process of the group number is repeated every $T_{\max }$ time.

The source node then sends out packets uniformly within the selected output time frame $T_{\text {out }}$. As $T_{\text {out }}$ is one of the G time frames, the packets which were buffered $T_{\max }$ time could be sent out in several $T_{\text {out }}$ frames. For example, if $T_{\text {max }}=4 T_{\text {min }}$ and $T_{\text {out }}=2 T_{\text {min }}$, i.e. $2 T_{\text {out }}=T_{\max }$, packets are buffered $T_{\max }$ time and sent out in two $T_{\text {out }}$ frames. The packets with group number 1 or 2 are sent out uniformly within the first $T_{\text {out }}$ frame, and the packets with group number 3 or 4 are sent out uniformly within the second $T_{\text {out }}$ frame.

Examples for the operations at the source node are given in Fig. 5 in which three kinds of different time frames $T_{1}, T_{2}$, and $T_{3}$ are chosen for the connection in which $T_{1}=2 T_{2}$ and $T_{2}=2 T_{3}$. Fig. 5 shows operations for two different arrival patterns and two different $T_{\text {out }}$ selections, $T_{\text {out }}=T_{1}$ for case (A) and $T_{\text {out }}=T_{2}$ for case (B). From the arrival pattern of case (A), packets $3,4,6$, and 9 are end packets of $T_{3}$, packets 4 and 9 are end packets of $T_{2}$, and packet 9 is the end packet

\begin{tabular}{|l|l|l|l|l|}
\hline End flag of $\mathbf{T}_{\mathbf{1}}$ & End flag of $\mathbf{T}_{\mathbf{2}}$ & $\ldots$. & End flag of $\mathbf{T}_{\mathbf{G}}$ & $\mathbf{T}_{\mathrm{G}}$ 's Group Number in $\mathrm{T}_{1}$ \\
\hline
\end{tabular}

Fig. 4. The flow control field of a MCF packet. 


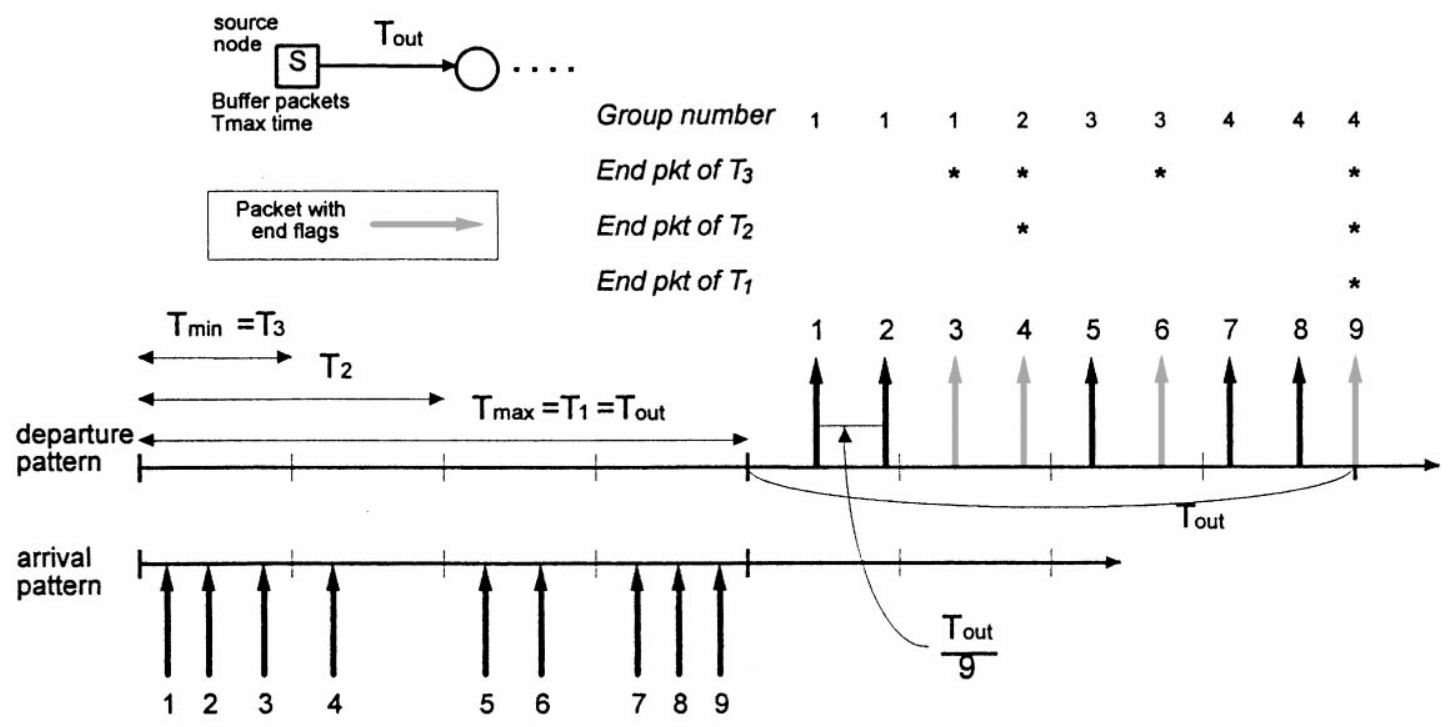

Case (A). Arriving pattern without empty groups. $\left(T_{\text {out }}=T_{1}\right)$

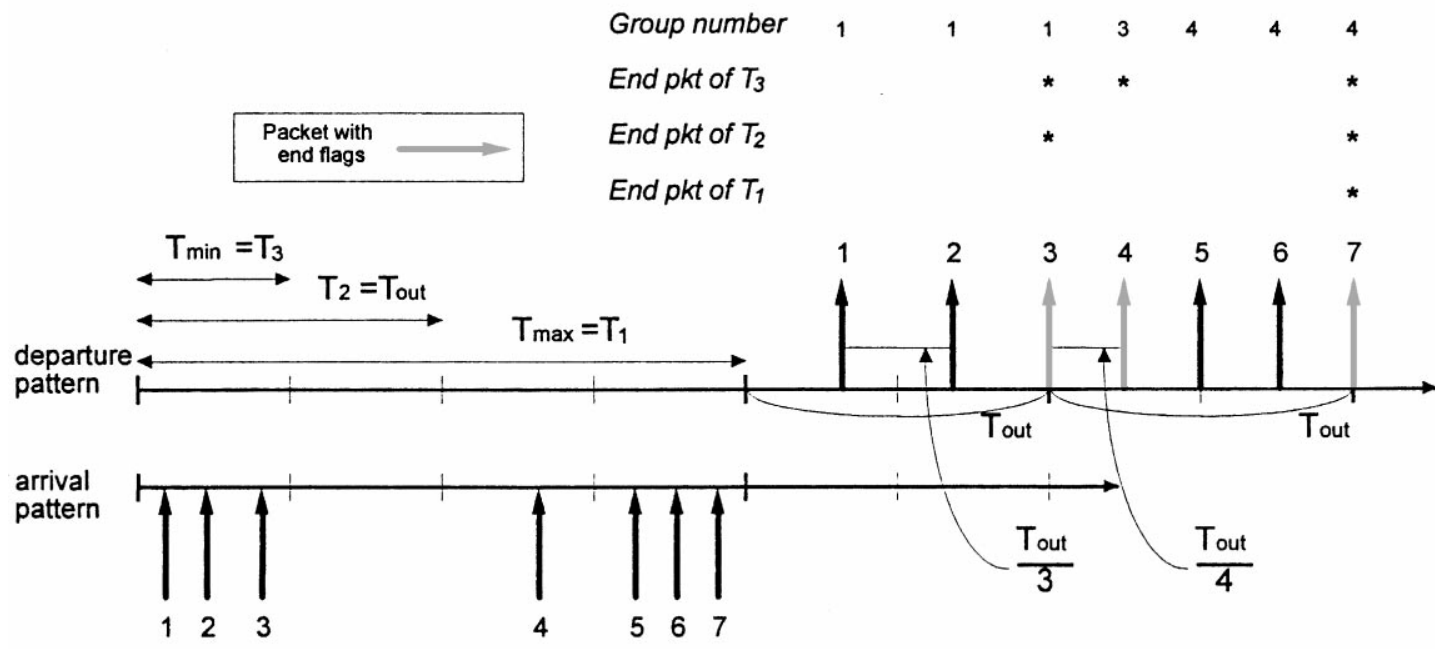

Case (B). Arriving pattern with an empty group. $\left(T_{\text {out }}=T_{2}\right)$

Fig. 5. Examples of the operations at the source node.

of $T_{1}$. An asterisk (*) is added above the end packets of the corresponding time frame. As packets 1,2 , and 3 arrived in the first $T_{\min }$ frame, the group number of these packets is 1 , packet 4 arrived in the second $T_{\min }$ frame, so its group number is 2 , etc. As $T_{\text {out }}$ in case (A) equals $T_{\max }$, all packets buffered are sent out uniformly within the next $T_{\text {out }}$ frame, i.e., the nine buffered packets are spaced by $T_{\text {out }} / 9$ in case (A) of Fig. 5.

For the arrival pattern of case (B), there is no packet arriving in the second $T_{\min }$ frame, and thus no packet has a group number of 2. As $T_{\text {out }}=T_{2}$ and $2 T_{\text {out }}=T_{\text {max }}$, the buffered packets are sent out in two consecutive $T_{\text {out }}$ frames. Packets with group number 1 or 2 (i.e. packets 1, 2, and 3) are sent out uniformly within the first $T_{\text {out }}$ frame, and packets
$4,5,6$, and 7 whose group number is either 3 or 4 are sent out uniformly within the second $T_{\text {out }}$ frame.

\subsubsection{MCF control at the intermediate nodes}

The end flags and group number carried by packets are used in changing the time frame length at the intermediate node. In this section, we only present the mechanism to change the time frame length instead of the selection of the time frame length. The strategy of selecting the time frame length is presented in section 3. One of following three situations may occur when an intermediate node serves a connection: (1) the length of the arrival time frame and that of the departure time frame are equal, (2) the length of the arrival time frame is a multiple of the 


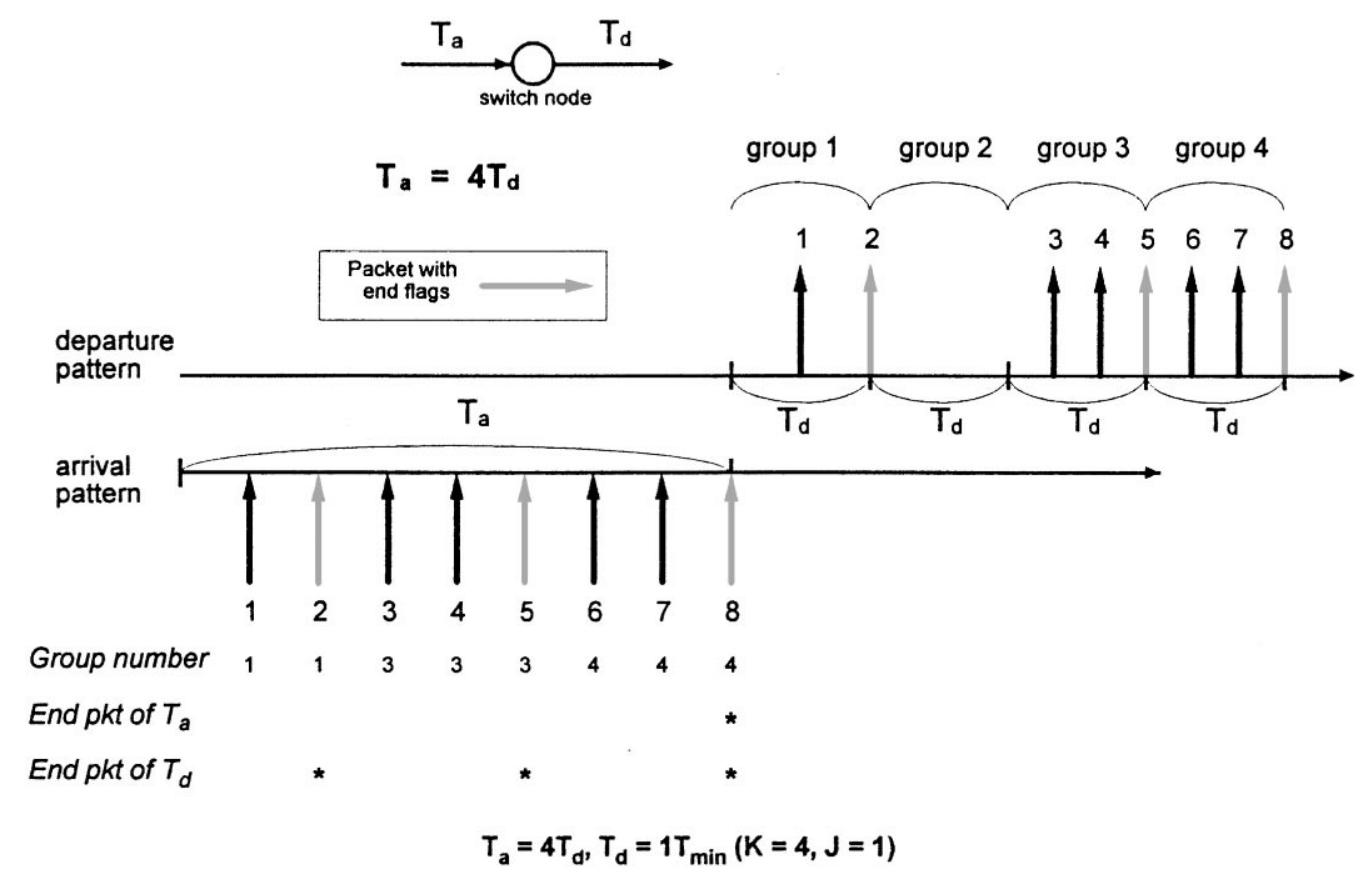

Fig. 6. Example of changing a larger time frame to smaller time frames.

length of the departure time frame, and (3) the length of the departure time frame is a multiple of the length of the arrival time frame. Operations of the intermediate nodes for situation (1) are the same as those in CF. Operations for situations (2) and (3) that require changing the time frame are described in the next section.

\subsubsection{Changing a time frame into smaller ones. Suppose} that the arrival time frame is $T_{\mathrm{a}}$ and the departure time frame is $T_{\mathrm{d}}$. We assume $T_{\mathrm{a}}=K^{*} T_{\mathrm{d}}, T_{\mathrm{d}}=J * T_{\min }, J, K \in N$. The intermediate node buffers the incoming packets until an end packet of $T_{\mathrm{a}}$ arrives. Next, the intermediate node starts $K$ consecutive $T_{d}$ frames. The node sends packets with group number $[1 \sim J]$ uniformly within the first $T_{\mathrm{d}}$ frame, and sends packet with group number $[J+1 \sim 2 J]$ uniformly within the second $T_{\mathrm{d}}$ frame, etc. In this way, the traffic pattern from the $T_{\mathrm{d}}$ 's point of view can be reconstructed in the departure frames of the intermediate node. An example is illustrated in Fig. 6.

In Fig. 6, the length of $T_{\mathrm{a}}$ is four times of the length of $T_{\mathrm{d}}$, so the packets buffered within a $T_{\mathrm{a}}$ frame are sent out in four consecutive $T_{\mathrm{d}}$ frames. The switch node decides which packet to be sent out according to the group number. Packets 1 and 2 with group number 1 are sent out uniformly in the first $T_{\mathrm{d}}$ frame. As there is no packet with group number 2, the second $T_{\mathrm{d}}$ frame is empty as shown in the figure. Packets 3,4 , and 5 with group number 3 are sent out in the third $T_{\mathrm{d}}$ frame, etc.

2.1.2.2. Changing time frames into a larger one. Similarly, we assume $K^{*} T_{\mathrm{a}}=T_{\mathrm{d}}, K \in N$. The intermediate node not only buffers the incoming packets until an end packet of $T_{\mathrm{d}}$ arrives, but also records the number of $T_{\mathrm{a}}$ frames which have arrived since the last end packet of $T_{\mathrm{d}}$. We assume the number is $Y(Y \leq K)$. When an end packet of $T_{\mathrm{d}}$ arrives, the intermediate node waits for $(K-Y)^{*} \mathrm{~T}_{\mathrm{a}}$ time, starts a new $T_{\mathrm{d}}$ frame, and sends buffered packets uniformly within the new frame. The purpose of the waiting time $(K-Y)^{*} T_{\mathrm{a}}$ is to buffer the arriving packets until the end of a $T_{\mathrm{d}}$ frame so that the timing of the arrival time frame is consistent with the timing of the departure time frame. Two examples are illustrated in Fig. 7 which includes two cases of $4 T_{\mathrm{a}}=T_{\mathrm{d}}$.

As the group number is not used in changing time frames into a larger one, the group number of each packet is not displayed in Fig. 7. In case (A) of Fig. 7, the switch node found that the end packet of $T_{\mathrm{d}}$, i.e. packet 8 , has arrived, and the number of $T_{\mathrm{a}}$ arrived is 4 , the switch node immediately starts a $T_{\mathrm{d}}$ frame and sends out the buffered packets within the frame. Case (B) of Fig. 7 shows the case in which the end packet of $T_{\mathrm{d}}$, packet 5 , has arrived while only $3 T_{\mathrm{d}}$ frames have arrived; therefore, the switch node waits for one extra $T_{\mathrm{a}}$ frame time and then starts a $T_{\mathrm{d}}$ frame to send out packets.

\subsection{Admission control of $M C F$}

The admission control mechanism is exercised before the connection is set up in order to prevent the network from being overloaded. The admission test of MCF is similar to that of CF except that the time frame length of a connection can be different on each link the connection passes, and the number of packets permitted to send out on each link may also be different. For connection $\mathrm{c}$ with the chosen time 


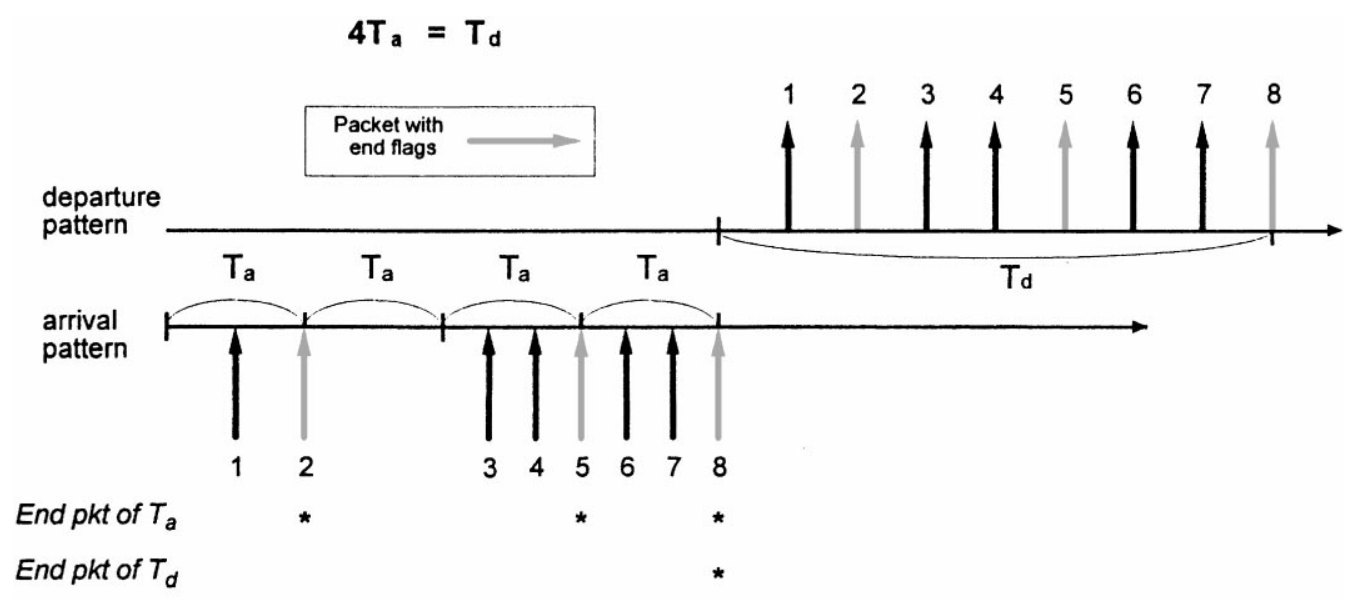

Case (A). $4 T_{a}=T_{d}(K=4), Y=4$.

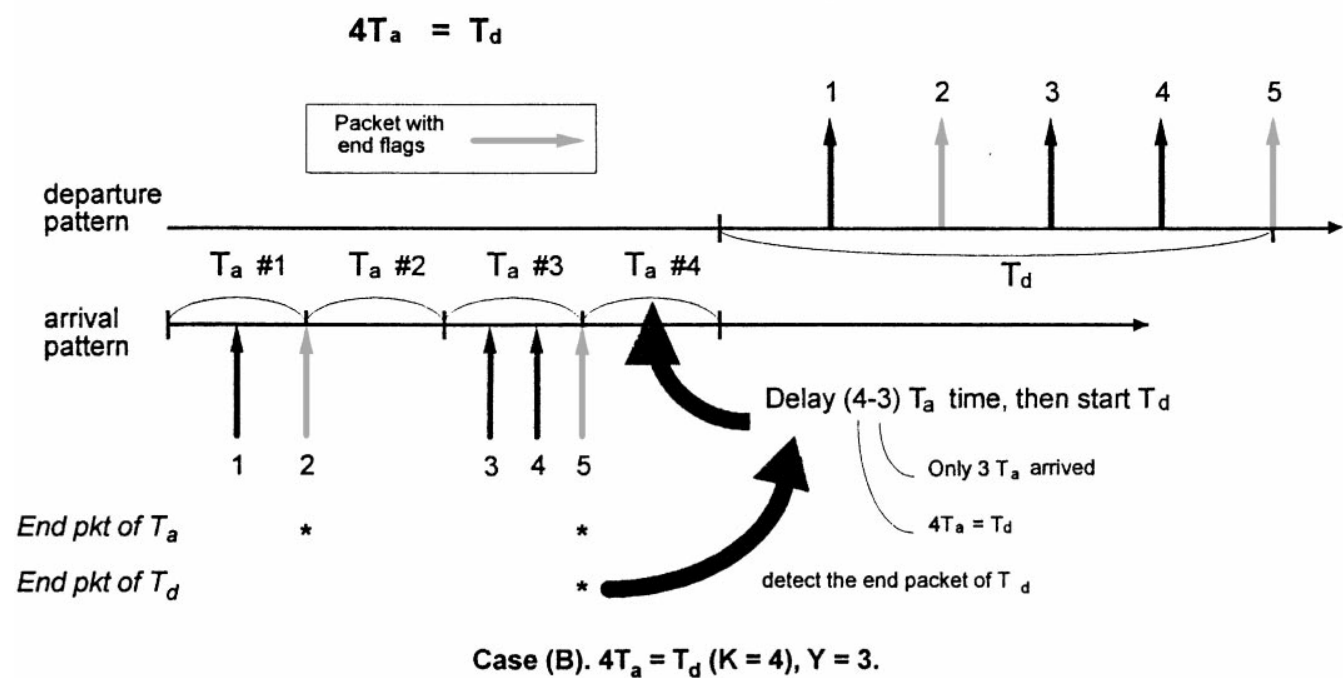

Fig. 7. Example of changing smaller time frames to a larger time frame.

frame $T_{j}$ for output link $j$, its contributed load on link $j$ will be $U_{j}=\left(M_{j} L\right) /\left(T_{j} C_{j}\right) \cdot M_{j}$ is the number of packets permitted to send on link $j$ during one time frame from connection $c, L$ is the number of bits in a packet, and $C_{j}$ is the capacity of the link (bits/s). Hence, for connection c to be admitted into the network, the following test should be exercised on each link it passes:

Utilization test: $\forall$ link $j$ on the path of connection $c$,

$\sum_{\forall \text { connections pass link } j} U_{j}<1$.

The utilization test guarantees that all links along the path are not saturated.

\subsection{End-to-end delay and jitters of MCF}

The end-to-end delay of a path is determined by the initial delay at the source node and by the time frame lengths of the input and output links at each intermediate node along the path. According to the operations of the switch nodes as described in section 2.1, the queueing delay bound at switch node $i$ is the larger value of the arrival time frame length $T_{\mathrm{a}}^{i}$ and the departure time frame length $T_{\mathrm{d}}^{i}$ That is, delay bound at a switch node $i$ is Max $\left(T_{\mathrm{a}}^{i}, T_{\mathrm{d}}^{i}\right)$. Therefore, if we define $D$ as the end-to-end delay bound, then

$D=T_{\text {initial }}+\sum_{i=1}^{H} \operatorname{Max}\left(T_{\mathrm{a}}^{i}, T_{\mathrm{d}}^{i}\right)+$ Propagation delay,

where $H$ is the switch count of the path, and $T_{\text {initial }}$ is the delay at the source node.

We define delay jitters as the maximum variation in delay experienced by packets in a single connection. For each switch node, the output traffic pattern of one connection will be reconstructed to be similar to its previous node. Hence, a similar output traffic pattern can be maintained throughout the network even if the time frame length is changed. So the jitters of the packets in MCF is between 
$-T_{\mathrm{d}}^{H}$ and $T_{\mathrm{d}}^{H}$, in which $T_{\mathrm{d}}^{H}$ is the departure time frame of the last switch node in the path. If we define $J$ as the maximum jitter (jitter bound), then

$J=2 T_{\mathrm{d}}^{H}$.

\subsection{Discussions and comparisons}

MCF inherits the characteristics of CF so that the techniques proposed in CF such as Delay Sending and Virtual Tag [17] can also be applied in MCF. Besides, MCF provides more flexibility than $\mathrm{CF}$ does. Basically, MCF is a generalized version of $\mathrm{CF}$.

Changing of the time frame length of a connection can either increase the statistical multiplexing gain for the connection or reduce the jitter bound by selecting a smaller time frame at the last switch node. However, changing the time frame length sometimes introduces a longer end-to-end delay.

As MCF is non work-conserving, it results in a lower link utilization than work-conserving schemes such as RealTime Virtual Clock [8, 9] and Generalized Processor Sharing $[19,20]$. However, MCF provides both delay bound and jitter bound. Many real-time applications, particularly those that are interactive, require a bound on jitter, in addition to a bound on delay. Note that certain applications such as noninteractive television and audio broadcasting require bounds on jitter but not delay. MCF therefore satisfies the requirement of these applications. The issue of jitter bound is not addressed in the control schemes like Real-Time Virtual Clock or Generalized Processor Sharing.

\section{Multicast connection setup for MCF}

\subsection{Basic concept}

The connection setup procedure of the time framing strategy determines the time frame length for each link along the path to satisfy the end-to-end delay bound of the connection. As CF allows only one time frame length for a connection, once the routing path (or the longest path for a multicast connection) is decided, we can derive the time frame length for each link as explained in section 1.

As for MCF, the flexibility of changing the time frame length gives us more freedom to select the time frame length for each link. As the end-to-end delay in MCF is determined by the initial delay $T_{\text {initial }}$ at the source node and the time frame length of each link as shown in Eq. (2), we discuss the relationship among $T_{\text {initial }}$ the time frame length, and the delay bound first.

$T_{\text {initial }}$ is the buffered time at the source node for setting the end flags in each packet. According to the control actions of MCF at the source node, which is presented in section 2.1.1, $T_{\text {initial }}$ must be larger than all time frame lengths adopted by the connection so that the framing information can be set for each packet. That is, once $T_{\text {initial }}$ is decided, the time frame length for each link of the connection can not be larger than $T_{\text {initial }}$. Therefore, we should assign a large value to $T_{\text {initial }}$ so that there is more room for the time frame length of each link to be enlarged.

However, a large $T_{\text {initial }}$ also enlarges the end-to-end delay, which means the possible room for the time frame length to be enlarged is reduced as the required delay bound must be satisfied. That is, if $T_{\text {initial }}$ is large, the time frame length for each link must be small in order to satisfy the requested delay bound such that the link utilization is reduced. From this point of view, $T_{\text {initia }}$ should not be too large.

Therefore, the computation of $T_{\text {initial }}$ in MCF is based on the concept of equally allocating the delay bound to the source node and switch nodes, which will be explained next. Once $T_{\text {initial }}$ is decided, the time frame length for each link can be decided. In general, there are three steps in connection setup procedure of MCF for unicast connections to determine $T_{\text {initial }}$ and each time frame length:

(1) Compute the minimum time frame length that each link can provide according to the traffic pattern and the current load of the link, and calculate the best end-to-end delay that the path can support.The minimum time frame length for link $i$ is denoted by MinTimeFrame link $i_{\text {. The }}$

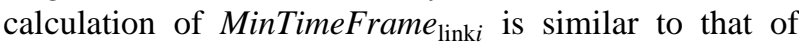
$\mathrm{S} \& \mathrm{G}$ and CF and is explained briefly. The traffic specification of a connection is provided by a set of $\left(r_{i}, T_{i}\right)$ smooth parameters, which means that during any interval of length $T_{i}$, the total arrived packets of the connection have no more than $r_{i} T_{i}$ bits. By examining the current load and the traffic pattern of the requested connection, each intermediate node can then determine the minimal frame size for the connection. The best end-to-end delay, denoted by BestTotalDelay, is computed by Eq. (2) in which MinTimeFrame $_{\text {linki }}$ is used for the time frame length and the term of $T_{\text {initial }}$ in the equation is replaced by the largest value of MinTimeFrame link1 $_{1}$, which is denoted by MaxofMinTimeFrames. That is,

$$
\begin{aligned}
& \text { BestTotalDelay }=\text { MaxofMinTimeFrames } \\
& +\sum_{i=1}^{H} \operatorname{Max}\left(\text { MinTimeFrame }_{\mathrm{link} i-1}, \text { MinTimeFrame }_{\mathrm{link} i} .\right.
\end{aligned}
$$

Note that MinTimeFrames is the basic term for $T_{\text {initial }}$.

(2) Determine the value of $T_{\text {initial }}$. RestDelay is defined as the difference between the requested delay bound and BestTotalDelay; that is, RestDelay represents the amount that can be allocated to the source node and switch nodes. We adopt the policy of equal distribution for allocating RestDelay, so the value of $T_{\text {initial }}$ equals the basic term MaxofMinTimeFrames plus RestDelay/SourceHopCount, where SourceHopCount is the number of source node and switch nodes, i.e. hop count plus one.

(3) Determine the time frame length of each link.The time frame length for each link can be relaxed instead 
(a)
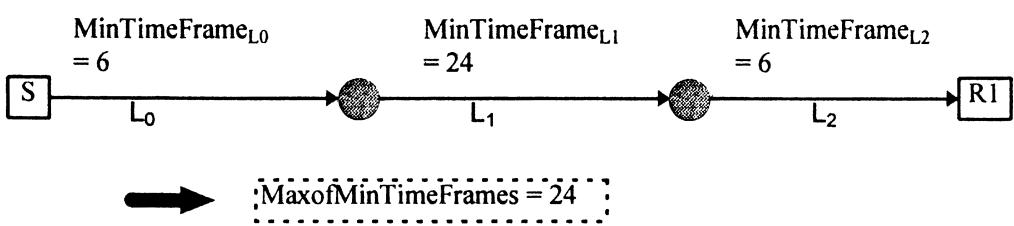

(b)

$$
\begin{aligned}
& \text { RestDelay }=300-(24+24+24)=228 \\
& \mathrm{~T}_{\text {initial }}=24+228 / 3=24+76=100-96
\end{aligned}
$$

(c)

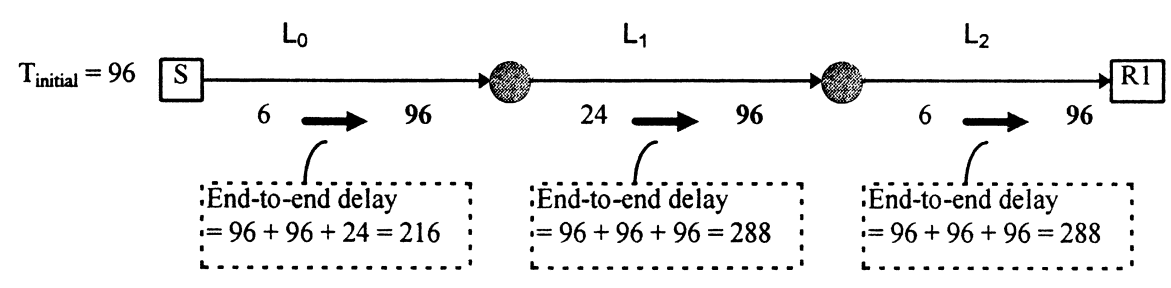

Fig. 8. An example for unicast connection setup in MCF.

of assigning MinTimeFrame link $i_{\text {To }}$ to link as long as the requested delay bound is satisfied. We call the process of enlarging the time frame length Relaxing.

We use the example in Fig. 8 to illustrate the connection setup procedure for a unicast connection. We assume that 7 time frame sizes 3, 6, 12, 24, 48, 96, and 192 can be used for the example, and the end-to-end delay bound and jitter

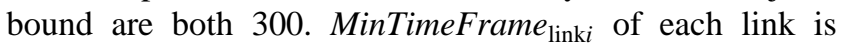
shown in part (a) of the figure. Part (b) shows the computa- tion of $T_{\text {initial, }}$ which equals Max of Min Time Frames $+($ RestDelay/SourceHopCount $)=24+(300-(24+$ $24+24) / 3)=100$. Hence 96 is assigned to $T_{\text {initial }}$. The relaxing process for the time frame length is illustrated in part (c) of the figure. For link $\mathrm{L}_{0}$ in the example, we can enlarge its time frame length up to 96 and the end-to-end delay bound is still satisfied as shown in part (c). Relaxing of links $\mathrm{L}_{1}$ and $\mathrm{L}_{2}$ is similar to that of link $\mathrm{L}_{0}$.

The connection setup procedure for multicast connections

DelayBound $=\mathbf{3 0 0}$, JitterBound $=\mathbf{3 0 0}$

7 frame sizes 3, 6, 12, 24, 48, 96, 192 can be used.

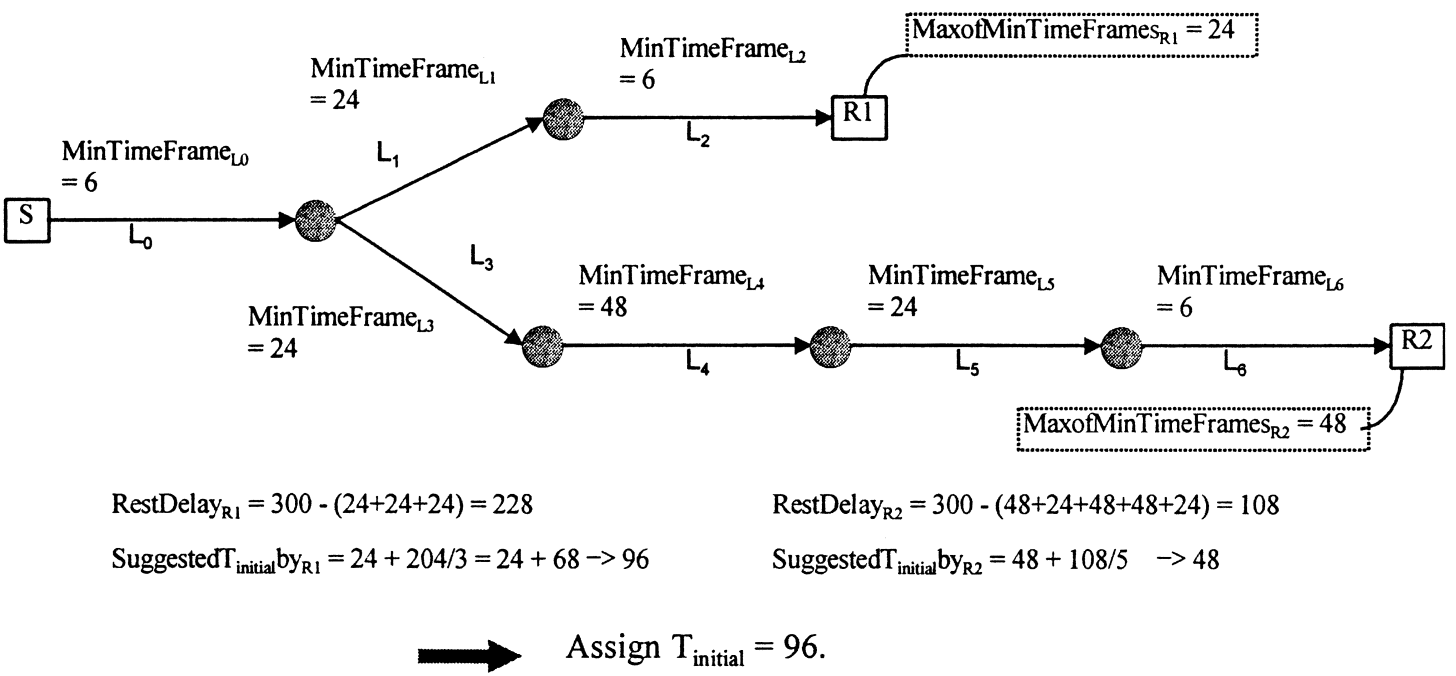

Fig. 9. An example of determining $\mathrm{T}_{\text {intial }}$ in MCF for a multicast connection. 


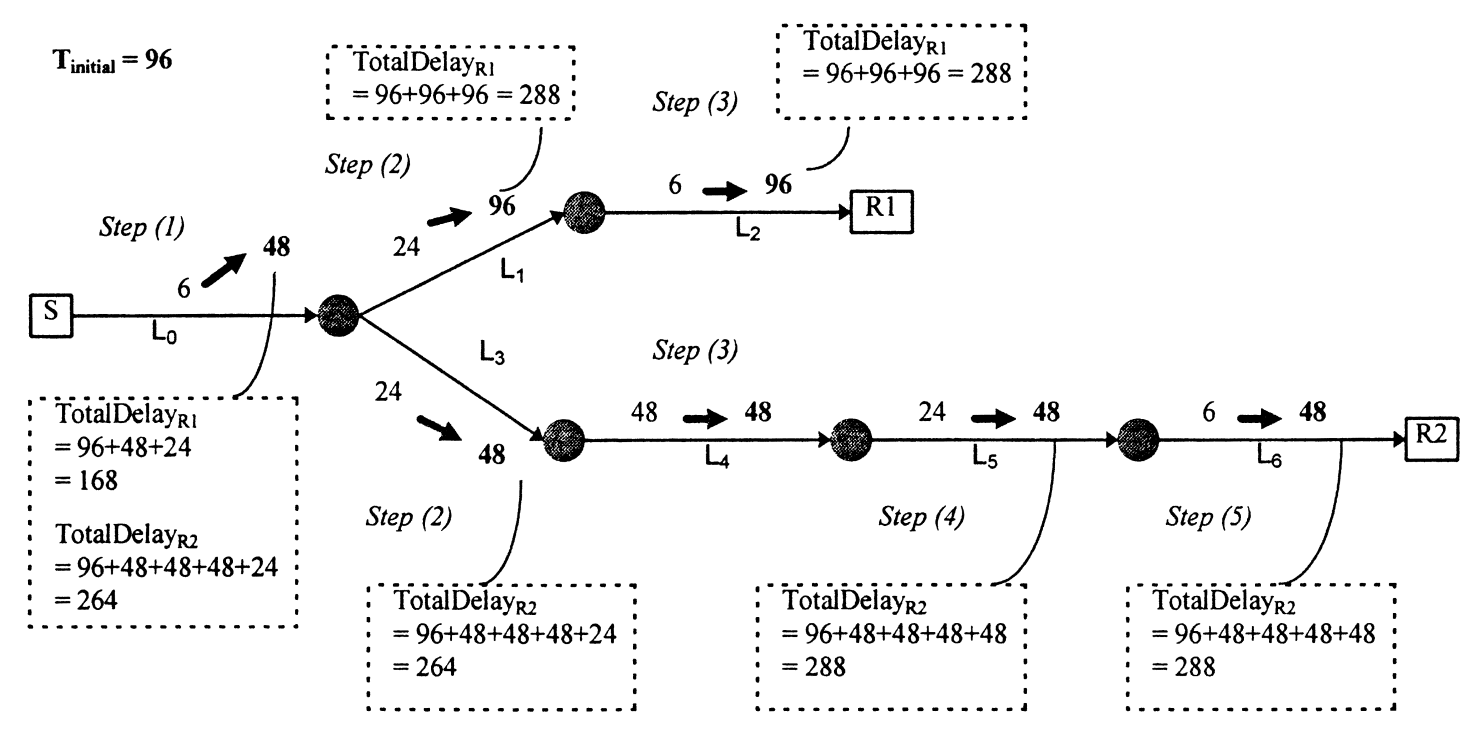

Fig. 10. The relaxing process of the example in Fig. 9.

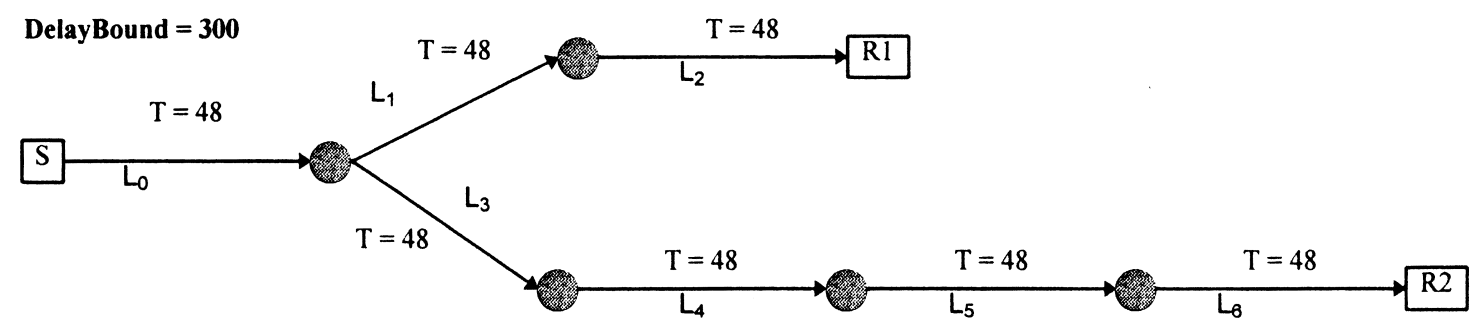

Fig. 11. An example of multicast connection setup in CF.

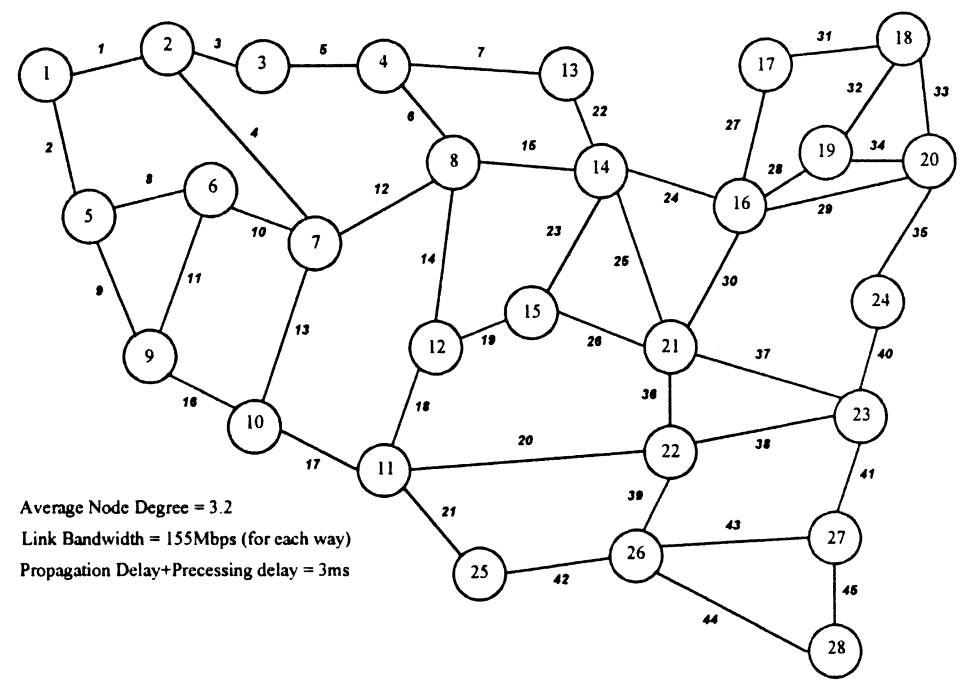

Fig. 12. The network topology for simulations.

is a little different from that of unicast connections as there are normally more than one receiver in a multicast connection. For multicast connection setup, first of all, each path from the sender to receivers is treated as an independent unicast connection, and each path computes the value of

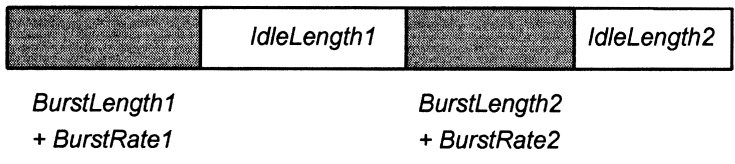

Fig. 13. The train model for traffic specifications. 


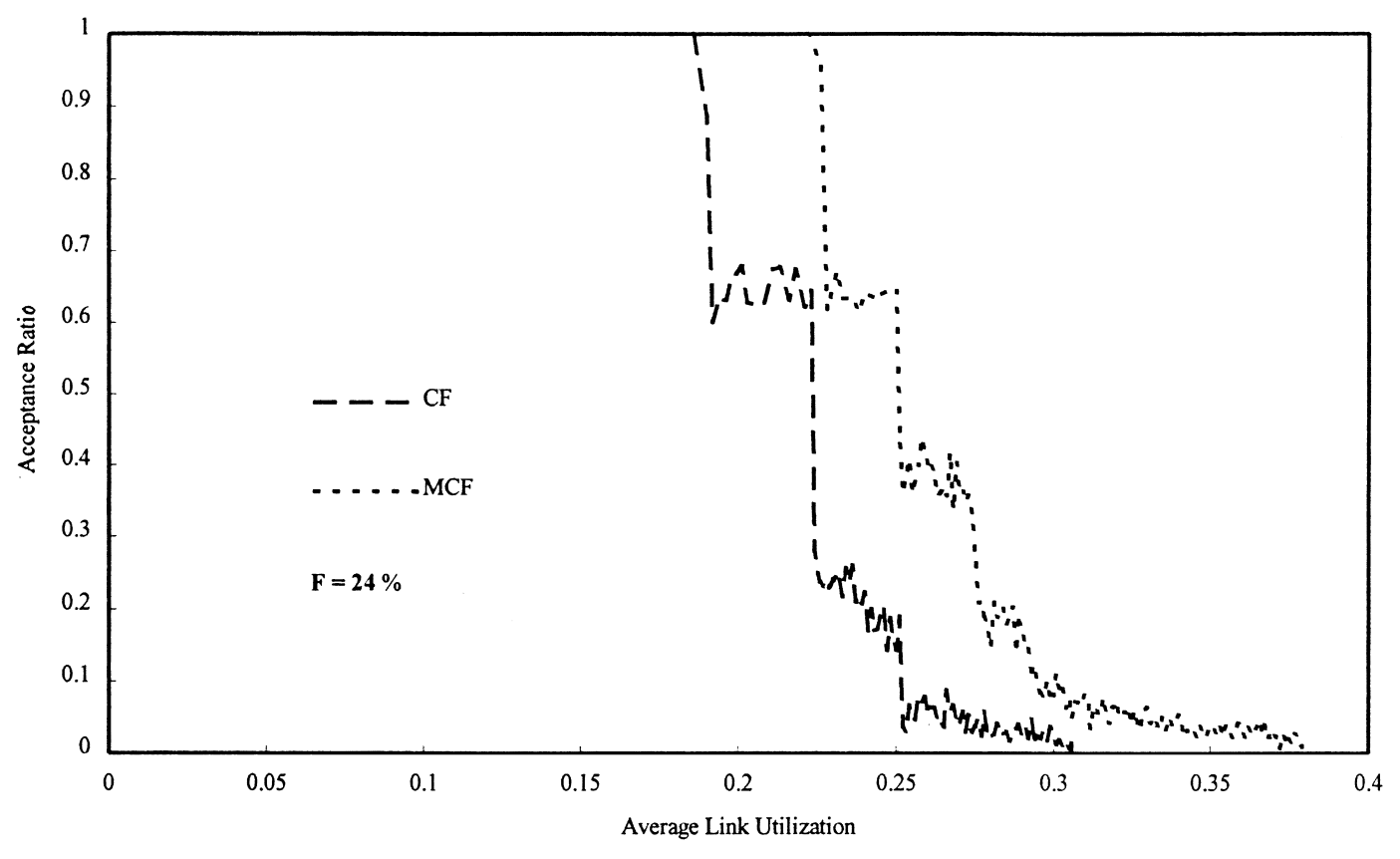

GroupSize $=2$, Traffic $=$ smooth, DelayBound $=300 \mathrm{~ms}$, JitterBound $=30 \mathrm{~ms}$

Fig. 14. Simulation result-1 for comparing MCF and CF.

$T_{\text {initial }}$ according to steps (1) and (2) mentioned above. The value is then suggested to the sender for determining the value of $T_{\text {initial }}$ for the multicast connection. The suggested value of $T_{\text {initial }}$ by the path from the sender to receiver $\mathrm{R}$ is denoted by Suggested $T_{\text {initial }} b y_{R}$. The sender then collects all values of Suggested $T_{\text {initial }} b y_{\mathrm{R}}$ and assigns the largest value of them to $T_{\text {intial }}$ under the constraint that the end-to-end delay bounds of all receivers are satisfied. The relaxing process is similar to that of unicast connections.

Fig. 9 shows an example of determining the initial delay $T_{\text {initial }}$ for a multicast connection in MCF. We also assume that 7 time frame sizes 3, 6, 12, 24, 48, 96, and 192 are used in the example, and the delay bound and jitter bound for all receivers are 300. MinTimeFrame linki $_{\text {in }}$ of each link is shown

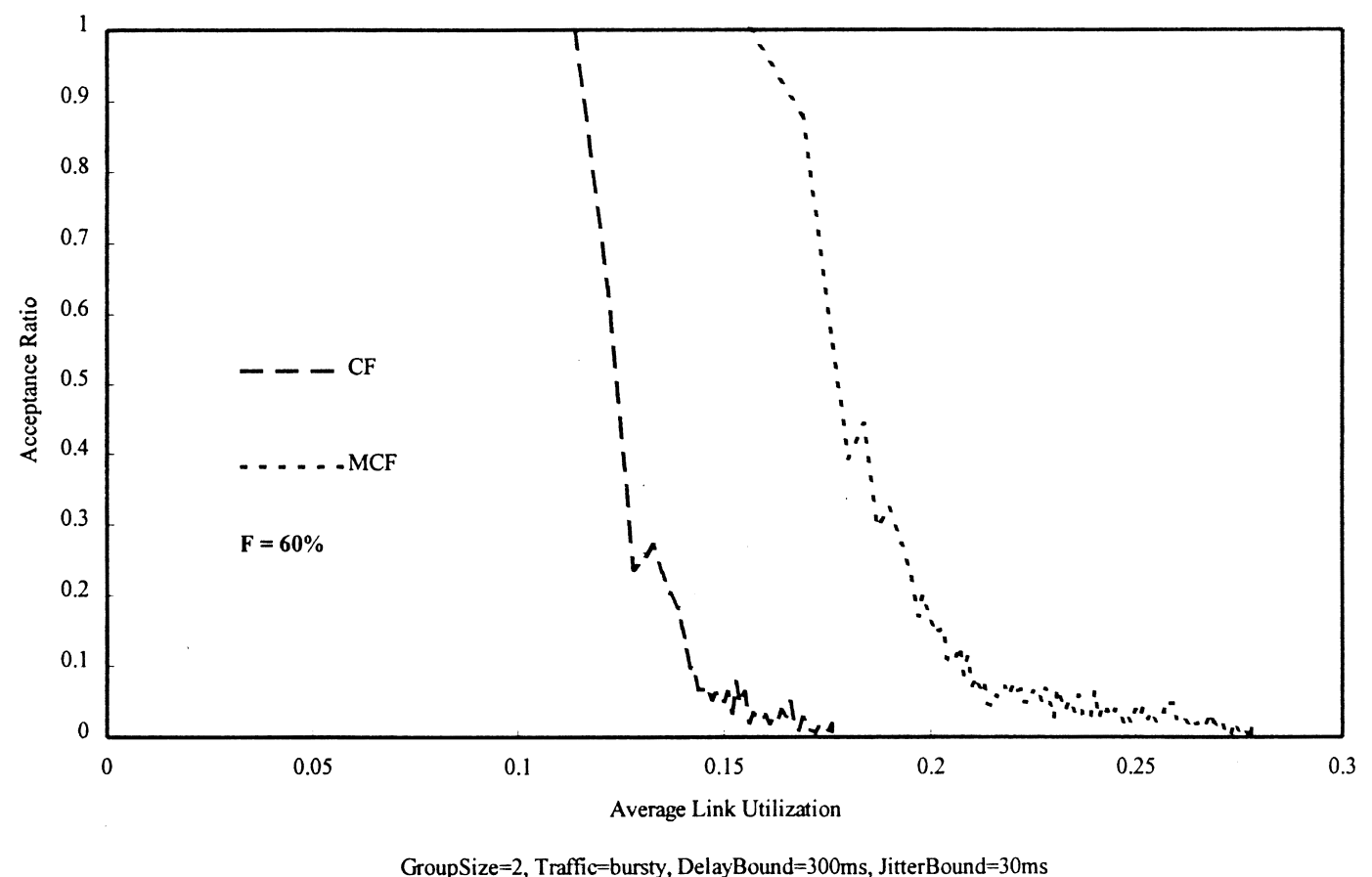

Fig. 15. Simulation result-2 for comparing $\mathrm{MCF}$ and $\mathrm{CF}$. 


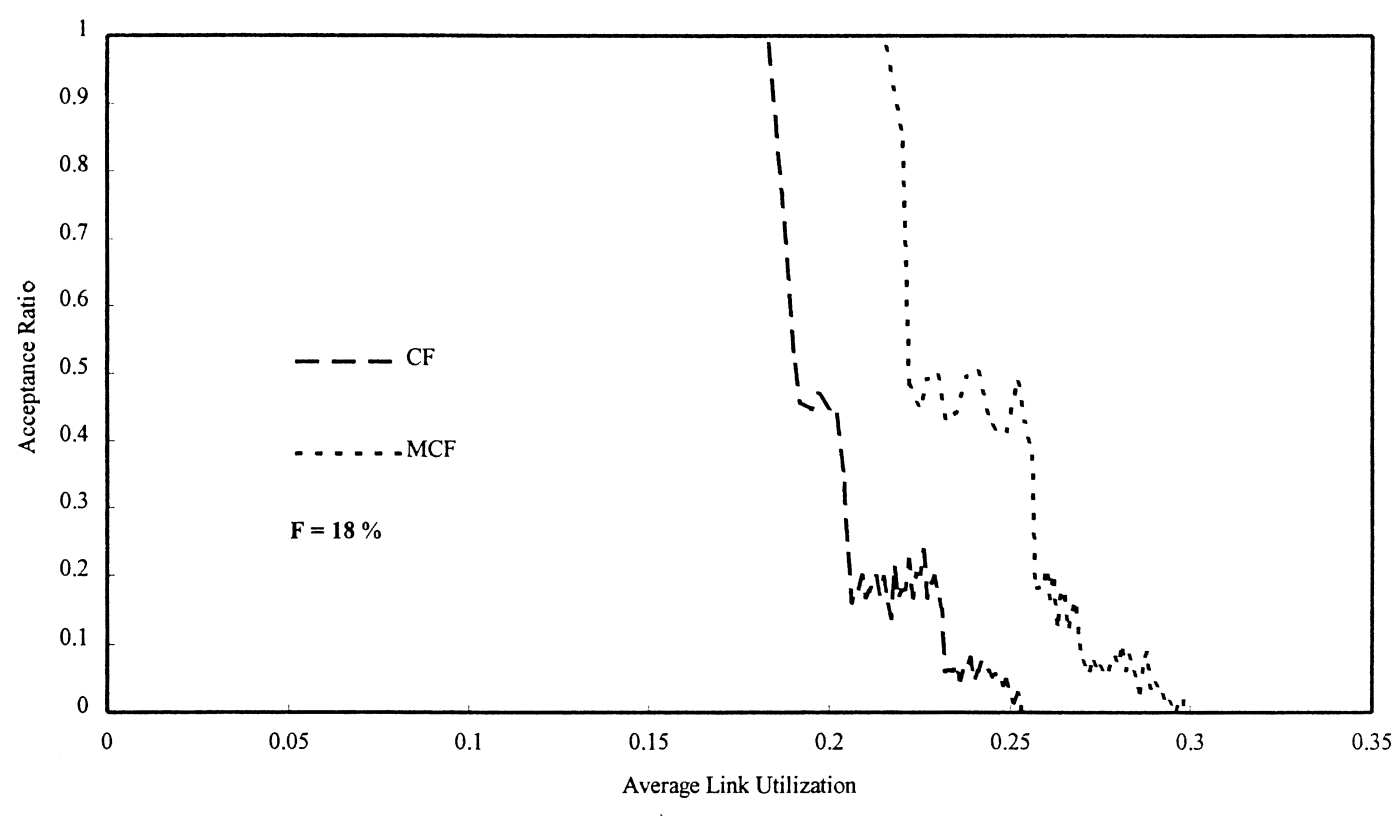

GroupSize $=4$, Traffic $=$ smooth, DelayBound $=300 \mathrm{~ms}$, JitterBound $=30 \mathrm{~ms}$

Fig. 16. Simulation result-3 for comparing MCF and CF.

in the figure. As shown in the figure,Suggested $T_{\text {initial }} b y_{\mathrm{R} 1}$

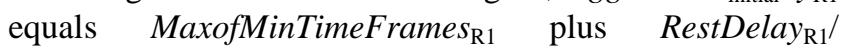
SourceHopCount $_{R 1}$, i.e., Suggested $T_{\text {initial }} b_{\mathrm{R} 1}=24+$ $228 / 3=24+76$, so we assign 96 to Suggested $T_{\text {initial }} b y_{R 1}$. Similarly, Suggested $T_{\text {initial }} b y_{\mathrm{R} 2}=48$. The value of $T_{\text {initial }}$ is the largest value of Suggested $T_{\text {initial }}$ by $y_{R}$ which does not make the end-to-end delay of all path exceed the requested delay bound. Therefore, we assign 96 to $T_{\text {initial }}$.

The relaxing process for the example in Fig. 9 is displayed in Fig. 10. Note that the total delay of path $\mathrm{S}-\mathrm{R} 1$ is computed as $T_{\text {initial }}+\operatorname{Max}\left(\right.$ TimeFrame $_{\mathrm{L} 0}$,

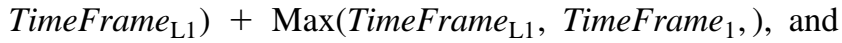
the total delay of path S-R2 is $T_{\text {initial }}+$ Max $\left(\right.$ TimeFrame $_{\mathrm{L} 0}$, TimeFrame $\left._{\mathrm{L} 3}\right)+\operatorname{Max}\left(\right.$ TimeFrame $_{\mathrm{L} 3}$, TimeFrame $\left._{\mathrm{L} 4}\right)+$ Max $_{\text {TimeFrame }} \mathrm{L} 4$, TimeFrame 55$)+$ Max (TimeFrame $_{\mathrm{L} 5}$, TimeFrame $\mathrm{L} 6$ ). Link $\mathrm{L}_{0}$ is first relaxed. Enlarging the time frame length of link $\mathrm{L}_{0}$ to 48 , the total delay of path S-R1 TotalDelay ${ }_{\mathrm{R} 1}$ becomes $(96+48+24)=168$, and the total delay of path S-R2 becomes $(96+48+48+48+24)=264$. If we enlarge the time frame length of link $\mathrm{L}_{0}$ to 96 , the total delay of path S-R2 becomes $(96+96+48+48+24)=312$ which violates the delay bound 300 . Therefore, the final time frame length of link $\mathrm{L}_{0}$ is 48 .

In step (2), link $\mathrm{L}_{1}$ and link $\mathrm{L}_{3}$ can be relaxed concurrently. Enlarging the time frame length of link $\mathrm{L}_{1}$ to 96 makes the total delay of path S-R1 288 as computed in the figure. Similarly, the time frame length of link $\mathrm{L}_{3}$ can be enlarged to 48 . The relaxing process of other links is similar to that of links $\mathrm{L}_{0}, \mathrm{~L}_{1}$, and $\mathrm{L}_{3}$ as presented above and the final time frame lengths are displayed in the figure.

Applying CF, instead of MCF, to the example in Figs. 9 and 10 , we can easily derive the unique time frame length of each link from the delay bound and the link count of the longest path. The time frame length using CF is displayed in Fig. 11. Comparing the final time frame length of each link by $\mathrm{MCF}$ in Fig. 10 with those by $\mathrm{CF}$ in Fig. 11, the time frame length of link $\mathrm{L}_{1}$ and $\mathrm{L}_{2}$ of Fig. 10 is larger than that of Fig. 11, which shows the flexibility advantage of MCF over CF.

In the next section, the algorithm of multicast connection setup for MCF is presented in detail. The algorithm is adopted in the simulation of performance evaluation in section 4 .

\subsection{Algorithm of multicast connection setup}

There are two steps for connection setup in MCF: (1) determining the initial delay and (2) relaxing the time frame for each switch node. The first way and the second way of connection setup determine the initial delay $T_{\text {initial }}$ of the connection, and the third way performs relaxing. Variables used in MCF are defined as follows:

[Variables used in MCF]:

\section{DelayBound}

the requested delay bound of all receivers.

JitterBound

the requested jitter bound of all receivers.

SourceHopCount ${ }_{\mathrm{R}}$

the number of the source node and switch nodes from the sender to receiver R, i.e. hop (switch) count plus one.

Link $_{0} \ldots$ link $_{H}$

the links from the sender to receiver $\mathrm{R}$, where $H$ is the 


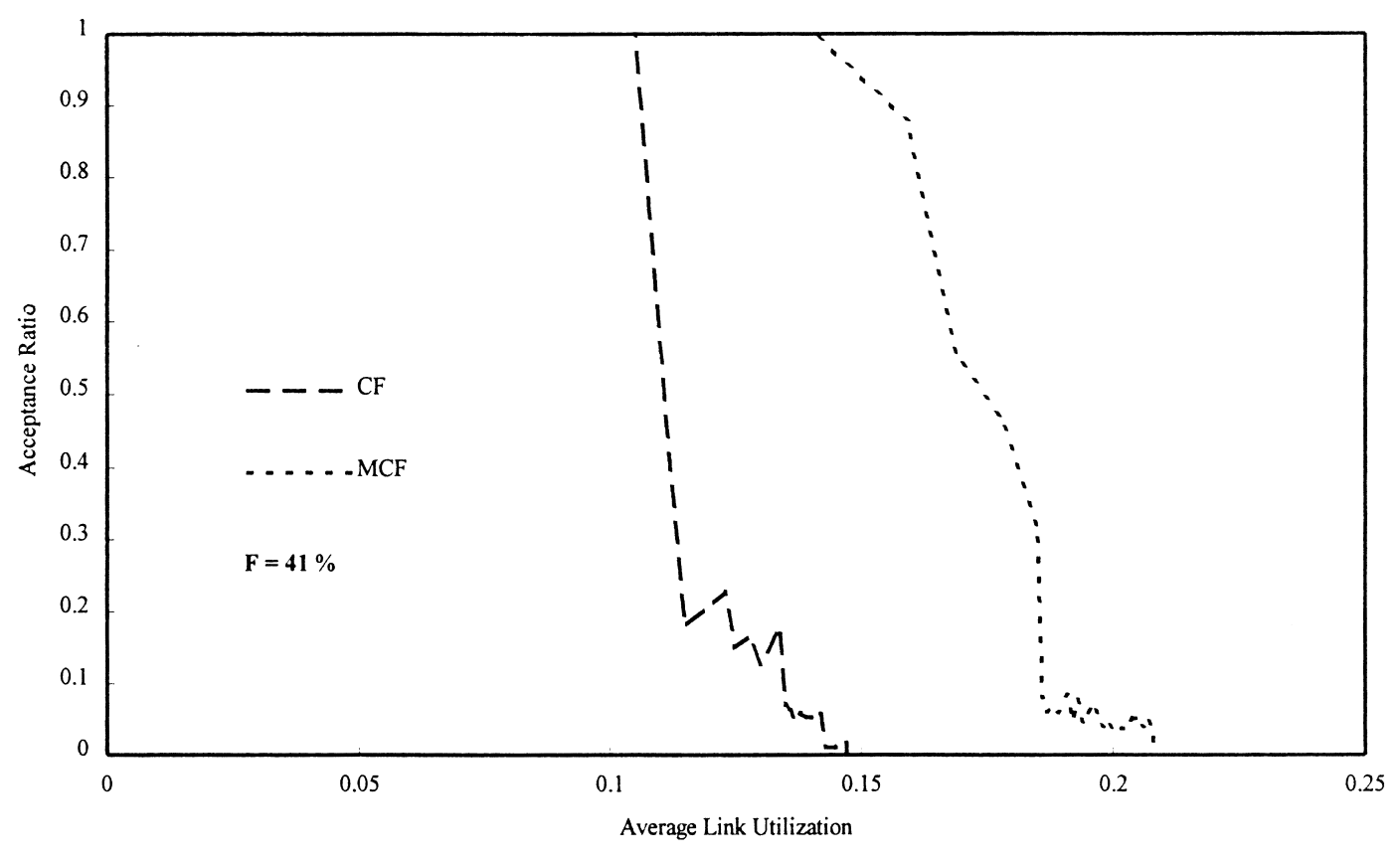

GroupSize $=4$, Traffic $=$ bursty, DelayBound $=300 \mathrm{~ms}$, JitterBound $=30 \mathrm{~ms}$

Fig. 17. Simulation result-4 for comparing MCF and CF.

switch count and link $\mathrm{k}_{0}$ is the link between the sender and the first switch node.

MinTimeFrame link $_{i}$

the minimum time frame that link $i$ can provide.

MaxofMinTimeFrames $_{\mathrm{R}}$

the largest value of MinTimeFrame link $i_{\text {in }}$ on the path from the sender to receiver $\mathrm{R}$.
BestTotalDelay $_{\mathrm{R}}$

the best total delay of the path from the sender to receiver R. Suggested $T_{\text {initial } \mathrm{by}_{\mathrm{R}}}$

the suggested value of $T_{\text {intial }}$ computed by receiver $\mathrm{R}$ in the first way.

RestDelay ${ }_{\mathrm{R}}$

the rest delay of the path from the sender to receiver $\mathrm{R}$.

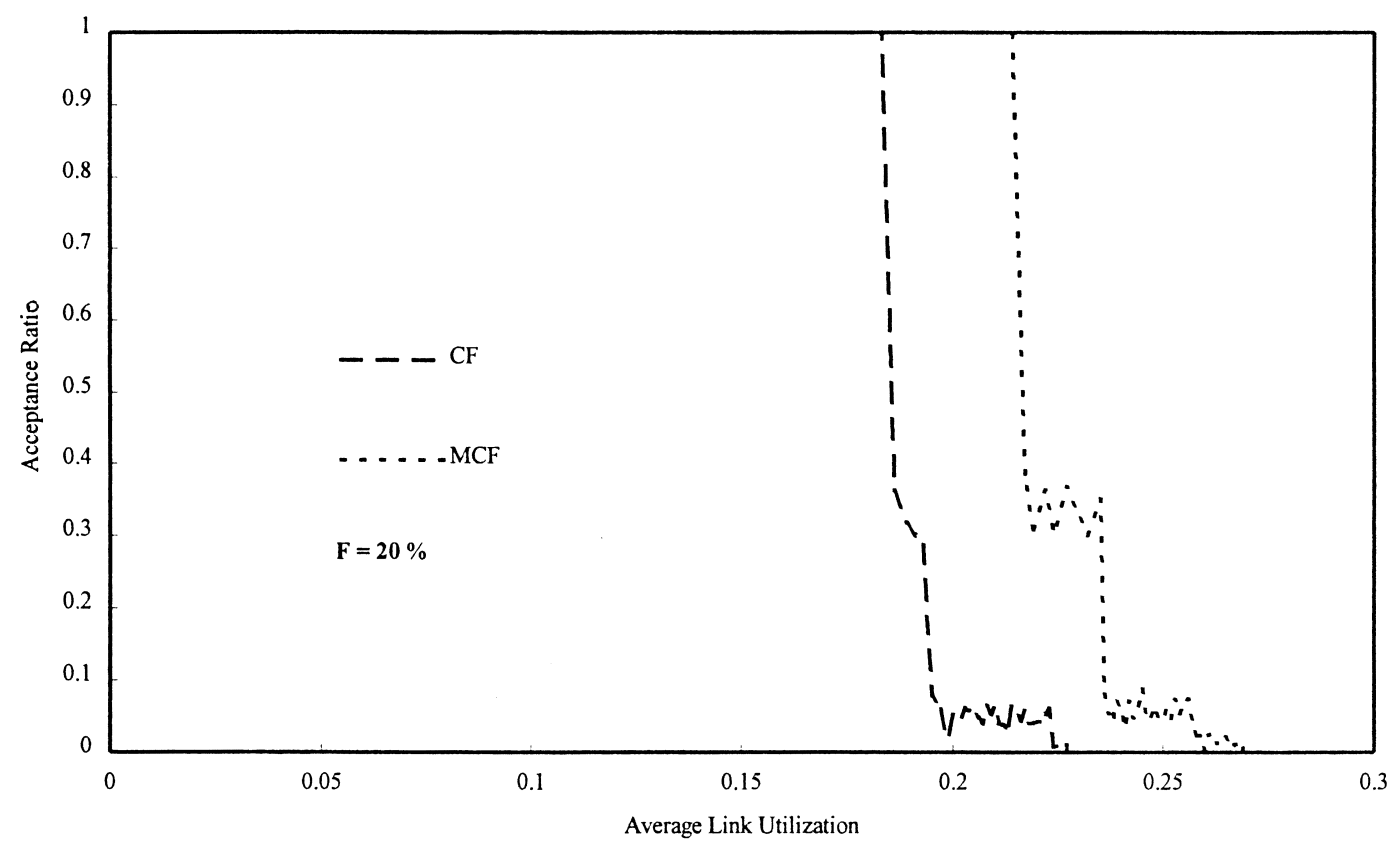

GroupSize $=8$, Traffic $=$ smooth, DelayBound $=300 \mathrm{~ms}$, JitterBound $=30 \mathrm{~ms}$

Fig. 18. Simulation result-5 for comparing MCF and CF. 


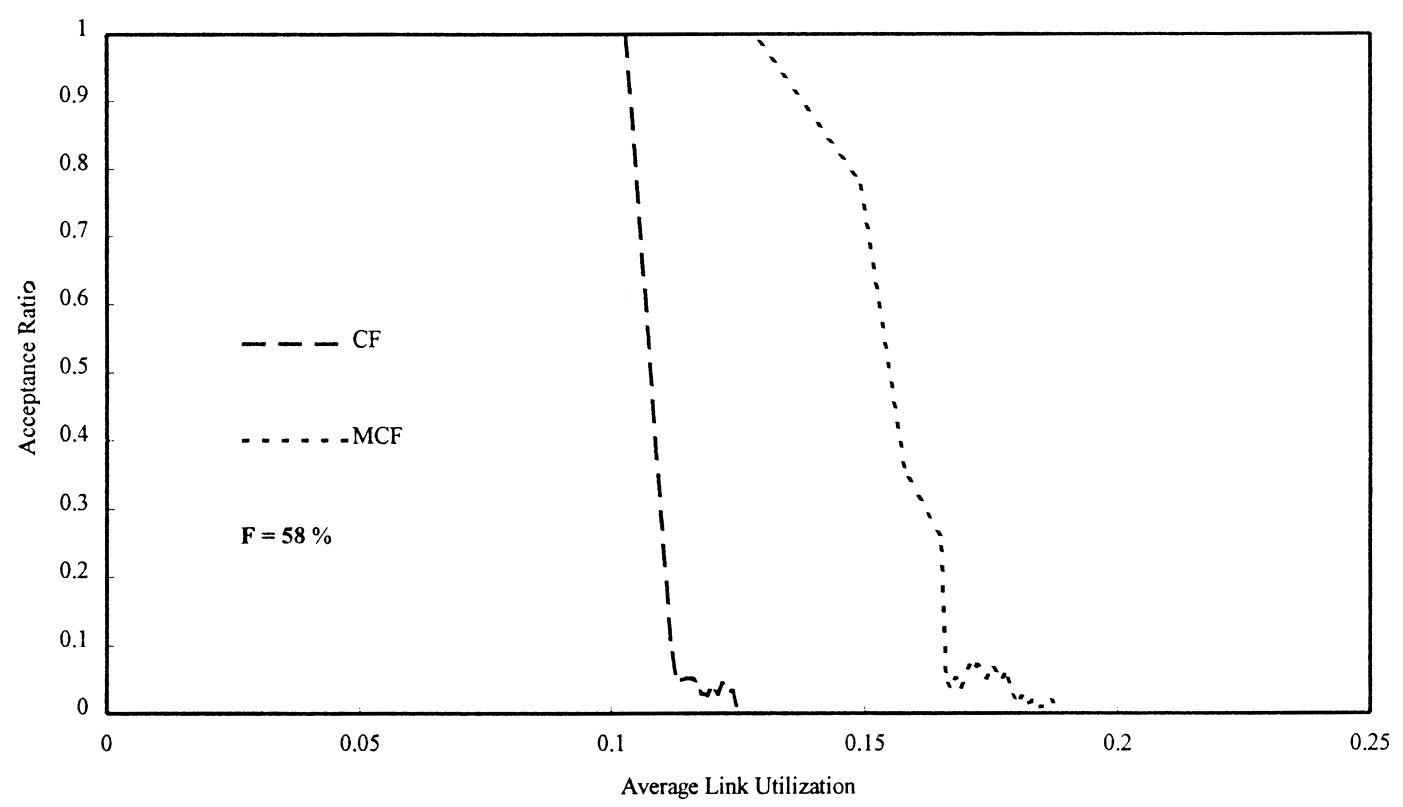

GroupSize $=8$, Traffic $=$ bursty, DelayBound $=300 \mathrm{~ms}$, JitterBound $=30 \mathrm{~ms}$

Fig. 19. Simulation result-6 for comparing MCF and CF.

$T_{\text {initial }}$ the initial delay chosen by the sender for the multicastconnection.

\subsubsection{1st way}

1. At each receiver $\mathrm{R}$, MaxofMinTimeFrames $\mathrm{R}_{\mathrm{R}}=$ Max (MinTimeFrame link $i_{\text {( }}$ )

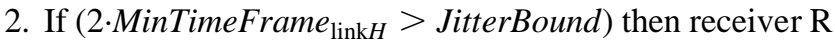
is rejected.where $\operatorname{link}_{H}$ is the last link of the path from the sender to receiver $\mathrm{R}$.

3. BestTotalDelay R $_{\mathrm{R}}=$ MaxofMinTimeFrams $_{\mathrm{R}}+$ $\sum_{i=1}^{H}$ Max $_{\text {MinTimeFrame }}$ link $i-1_{1}$, MinTimeFrame $\left._{\text {link } i}\right)$

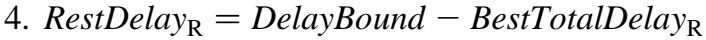

If $\left(\right.$ RestDelay $\left._{R}<0\right)$ then receiver $\mathrm{R}$ is rejected.

5. Suggested $T_{\text {initial }}$ by ${ }_{\mathrm{R}}=$ MaxofMinTimeFrames $_{\mathrm{R}}+$ RestDelay $_{\mathrm{R}} /$ SourceHopCount $_{\mathrm{R}}$

\subsubsection{2nd way}

The sender collects MinTimeFrame linki $_{\text {and }}$ Suggested $\mathrm{T}_{\text {initial }}$ by $\mathrm{H}_{\mathrm{R}}$.

The initial delay $T_{\text {initial }}$ is assigned as the largest value of Suggested $\mathrm{T}_{\text {initial }} \mathrm{by}_{\mathrm{R}}$ under the condition that the total end-toend delay $\leq$ DelayBound, $\forall$ receiver R.

\subsubsection{3rd way: [Relaxing]}

We enlarge the time frame length of each link on the path according to the following three constraints in which the new time frame length of the link being relaxed is denoted by NewTimeFrame.

1. NewTimeFrame $\leq T_{\text {initial }}$
2. After updating the new time frame length, the end-to-end delay of the path which the link is on can not be larger than the delay bound, i.e., $T_{\text {initial }}+\sum_{i=1}^{H}$ $\operatorname{Max}\left(\right.$ TimeFrame $_{\text {link } i-1}$, TimeFrame $\left._{\text {link } i}\right) \leq$ DelayBound, $_{\text {, }}$ where TimeFrame $_{\text {link } i}$ is the time frame length of link $i$. The relaxing process continues until all links on the path are relaxed.

3. If the time frame is for the last link of the path to receiver $\mathrm{R}$, then the following condition must be satisfied:

\section{NewTimeFrame $\leq$ JitterBound}

\section{Performance evaluation}

Some simulation results are presented in this section to compare $\mathrm{MCF}$ with $\mathrm{CF}$ in performance. The experimental environment including the network topology, the traffic model, and the way to construct a multicast tree are described next.

\subsection{Experimental environment}

The network topology used in the simulation is shown in Fig. 12 in which the average degree of node is 3.2, the bandwidth of each link is assumed to be $155 \mathrm{Mbps}$ for each direction, and the bandwidth of links to the end hosts, which are not displayed in the figure, is assumed to be $620 \mathrm{Mbps}$. Note that only switch nodes are displayed in Fig. 12, and the end hosts are not displayed in the figure. We adopt the train model as illustrated in Fig. 13 to specify the traffic pattern. The traffic pattern can be described by six parameters: BusrtLength1, BurstRate1, IdleLength1, BurstLength2, BurstRate2, and IdleLength2. Two sets of the 


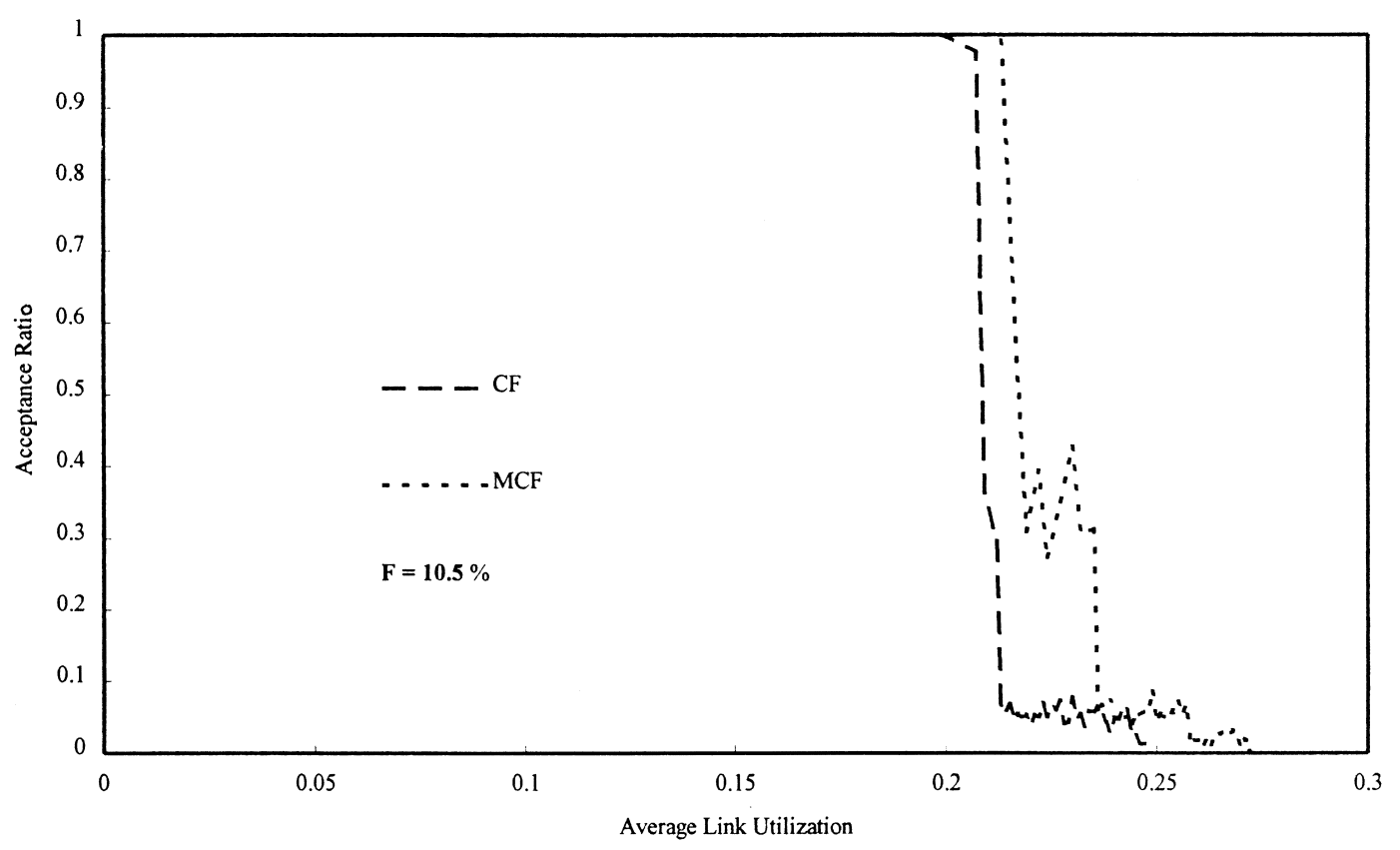

GroupSize $=8$, Traffic $=$ smooth, DelayBound $=300 \mathrm{~ms}$, JitterBound $=300 \mathrm{~ms}$

Fig. 20. Simulation result-7 for comparing MCF and CF.

parameters, one for smooth traffic and the other for bursty traffic are used in the simulations:

1. Smooth traffic: BusrtLength $1=30 \mathrm{~ms}$, BurstRate $1=$ $8 \mathrm{KBytes} / \mathrm{sec}, \quad$ IdleLength $1=30 \mathrm{~ms}, \quad$ BurstLength $2=$ $20 \mathrm{~ms}, \quad$ BurstRate $2=4 \mathrm{KBytes} / \mathrm{sec}, \quad$ IdleLength $2=$ $20 \mathrm{~ms}$.

2. Bursty traffic: BusrtLength $1=20 \mathrm{~ms}$, BurstRate $1=$ $48 \mathrm{KBytes} / \mathrm{sec}$, IdleLength $1=20 \mathrm{~ms}$, BurstLength $2=$ $30 \mathrm{~ms}, \quad$ BurstRate $2=4 \mathrm{KBytes} / \mathrm{sec}, \quad$ IdleLength $2=$ $30 \mathrm{~ms}$.

The switch nodes for the sender and receivers of a multicast connection are randomly and uniformly selected from the 28 nodes in the network. The number of receivers is determined by the group size of the connection. Once the sender and receivers of a connection are decided, the Shortest Path Tree (SPT) [18] algorithm is adopted to construct a multicast routing tree for each connection. SPT algorithm in the simulation first finds the shortest delay paths from the sender to all receivers, then the algorithm combines the paths found into a multicast tree.

The packet size used in the simulation is 53 bytes and the sum of packet processing time and propagation delay on each link is assumed to be $3 \mathrm{~ms}$. Each switch node selects the time frame of a connection on an output link from the following 7 time frame lengths: $3 \mathrm{~ms}, 6 \mathrm{~ms}, 12 \mathrm{~ms}, 24 \mathrm{~ms}$, $48 \mathrm{~ms}, 96 \mathrm{~ms}$, and $192 \mathrm{~ms}$.

\subsection{Simulation results and discussions}

The criterion to evaluate the performance in the simulation is the acceptance ratio of connection setup requests with respect to the corresponding average link utilization. The acceptance ratio is calculated as that the number of successful connection setup divided by the number of total connection requests. A successful connection setup requires the delay bound and jitters bound of all receivers in a connection to be satisfied. The simulation program computes and records the acceptance ratio for every 300 connection requests, and the simulation will stop when there are more than 600 consecutive connection setup requests failures. In this case, the network is defined as saturated.

To compare MCF with $\mathrm{CF}$, we define an improvement factor $F$ as the ratio of increased link utilization of MCF over CF when the network is saturated. That is,

$F=$

$\frac{\text { link utilization using } \mathrm{MCF}-\text { link utilization using } \mathrm{CF}}{\text { link utilization using } \mathrm{CF}}$.

1. Figs. 14 and 15 show results of group size 2 ( 2 receivers) with different traffic patterns (smooth, bursty) for delay bound $300 \mathrm{~ms}$ and jitter bound $30 \mathrm{~ms}$. Results of group sizes 4 and 8 are shown in Figs.16,17 and Figs.18,19 respectively. MCF obtains a significant performance improvement over $\mathrm{CF}$ when connections require a tight jitter bound $(30 \mathrm{~ms})$ by comparing Figs. 14-19. The reason is that the time frame length of each link on the path in CF is the same and is limited by the jitter bound, hence a small jitter bound forces the frame length to be also small and thus reduces the link utilization. Instead, 


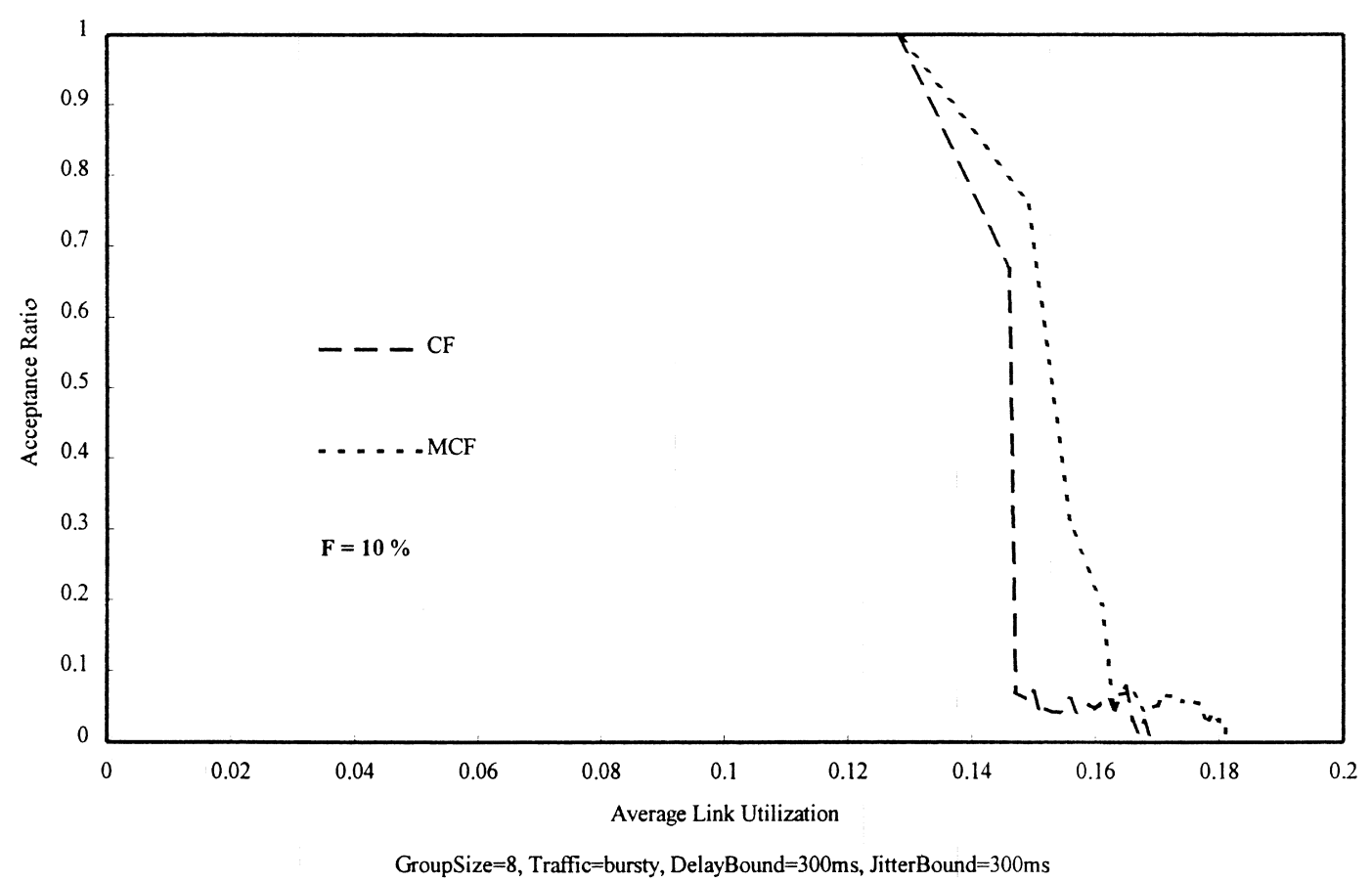

Fig. 21. Simulation result- 8 for comparing MCF and CF.

only frame length of the last link of a connection path is limited by the jitter bound in MCF, and the ability of changing the time frame length releases the limitation of time frame lengths of other links on the path. Therefore, link utilization is improved significantly in MCF as shown in the simulation.

2. Figs. 20 and 21 display the simulation results of delay bound $=$ jitter bound $=300 \mathrm{~ms}$. From these figures, the performance improvement factor $\mathrm{F}$ decreases to $10 \%$ while the connection requires a looser jitter bound. By comparing the curves of MCF in Fig. 18 with that in Fig. 20 and Fig. 19 with Fig. 21, there is no significant difference in the average link utilization between cases of jitter bound equals $30 \mathrm{~ms}$ and jitter bound equals $300 \mathrm{~ms}$. However, CF obtains better link utilization in the case of jitter bound equals $300 \mathrm{~ms}$ than that of jitter bound equals $30 \mathrm{~ms}$, since the time frame length in $\mathrm{CF}$ is only limited by the delay bound and the longest path instead of the jitter bound. A better link utilization of CF under a looser jitter bound implies that the jitter bound may affect the link utilization significantly, while the performance of MCF is almost not affected by a tighter jitter bound as mentioned earlier.

3. As we can see from the simulation results of smooth and bursty traffic patterns, the improvement of MCF over CF under bursty traffic, as displayed in the figures, is more significant than those under smooth traffic. The reason is that a larger time frame length can obtain more multiplexing gain for bursty traffic pattern. Although the performance of MCF under bursty traffic also degrades, the improvement percentage of MCF over $\mathrm{CF}$ is increased.
4. In order to inspect the impact of hop count on the performance, we introduce the constraint of hop count for the multicast group so that the sender and receivers of a group do not spread wide over the network. More specifically, hop count constraint $X$ means the sender and receivers in the same multicast group can only locate at a distance of hop count $<=X$. For example, the sender attached to switch node 1 can only have receivers attached to switches 2, 3, 5, 6, 7, 9 in Fig. 12 if $X=3$. Note that the hop count constraint only limits the selection of sender and receivers, the path length of the final route for a multicast group may exceed the hop count constraint as the route finding algorithm tries to search for a smallest delay path from the sender to each receiver. The acceptance ratio for the case of $X=3$ is displayed in Fig. 22. By comparing Fig. 15 with Fig. 22, the acceptance ratio and average link utilization in MCF under $X=3$ increases much more than that without hop count constraint. This is reasonable since more delay can be allocated to each link under the condition $X=3$.

5. All figures of the simulation results show that the phenomenon of sudden drop of the acceptance ratio always happens in the figures. This consequence is caused by the route finding algorithm of the simulation. The route finding algorithm tries to find a path with the minimum total delay from the sender to each receiver, and combines the paths found for all receivers. Thus when the network load is light, the acceptance ratio for connection requests is always $100 \%$ which means no connection request is rejected. Once a connection request is rejected from setting up, it is very likely that some links' resources are almost fully allocated, and that 


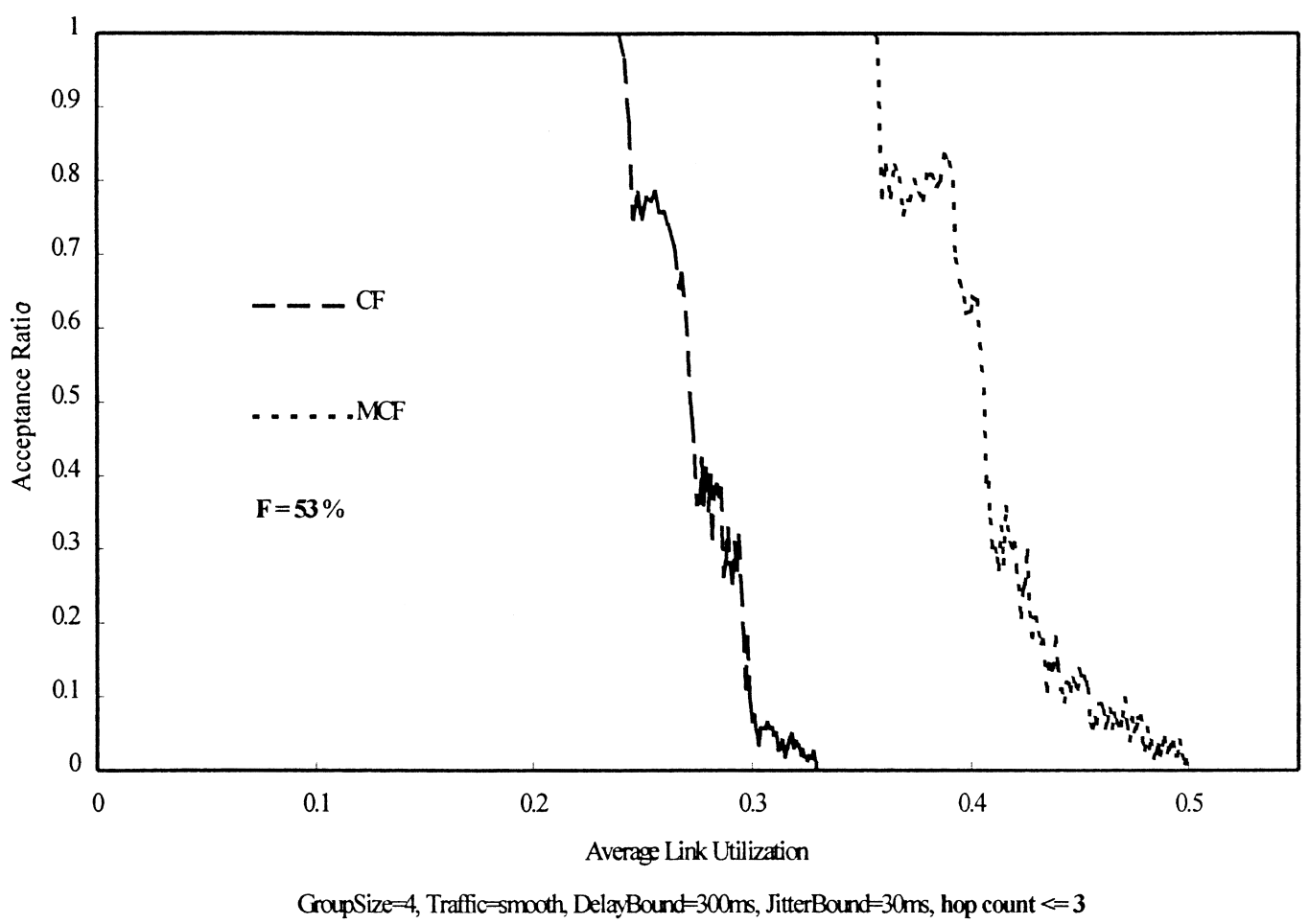

Fig. 22. Acceptance ratio and link utilization under hop count $<=3$.

causes the network topology to be divided into several parts. The following connection requests will be rejected if the sender and receivers of the connection are located in different parts. Therefore, the acceptance ratio drops suddenly. The phenomenon of sudden degradation continues when more links get saturated and divide the network into more isolated parts until the whole network is saturated.

\section{Conclusion}

Considering the characteristics of multicast transmissions and the time framing strategy, MCF removes the dependency of the time frame lengths of links along the path of a connection by allowing the time frame length to be changeable. By so doing, simulation results show that up to $60 \%$ link utilization improvement can be achieved in some cases compared with those using only Continuous Framing strategy. Moreover, as $\mathrm{MCF}$ is a generalized form of $\mathrm{CF}$, thus all techniques associated with $\mathrm{CF}$ such as delay sending and virtual tag can also be adopted by MCF to further improve the link utilization. In summary, major contributions of this article are listed below.

1. MCF provides bounded end-to-end delay/jitter and loss free transmissions as in $\mathrm{CF}$. Moreover, MCF allows changing of the time frame length such that the link utilization is increased.

2. The link utilization of MCF for connections requiring tighter jitter bounds is enhanced even more significantly over CF as shown in the simulations. Similarly, MCF performs much better than $\mathrm{CF}$ when the traffic pattern is bursty.

3. MCF only introduces a small overhead during the connection setup phase and a small overhead during the data transmission phase; hence, MCF is suitable for real-time applications over high-speed networks.

\section{References}

[1] H Zhang, S Keshav, Comparison of rate based service disciplines, ACM SIGCOMM (1991) 113-121.

[2] D.D. Clark, S. Shenker, L Zhang, Supporting real-time applications in an integrated services packet network: architecture and mechanism, SIGCOMM (1992) 14-26.

[3] M. Sidi, W.-Z. Liu, I. Cidon, I. Gopal, Congestion control through input rate regulation, IEEE Trans. on Comm. (1993) 471-477.

[4] C.M. Aras, J.F. Kurose, D.S. Reeves, H. Schulzrinne, Real-time communication in packet-switched networks, in: Proceedings of IEEE, Jan. 1994, pp.122-139.

[5] A. Campbell, G. Coulson, D. Hutchison, A quality of service architecture, ACM SIGCOMM (1994) 6-27.

[6] A. Demers, S. Kashav, S. Shenker, Analysis and simulation of a fairing-queueing algorithm, ACM SIGCOMM (1989) 1-12.

[7] C.R. Kalmanek, H. Kanakia, Rate controlled servers for very highspeed networks, GLOBECOM (1990) 30-39.

[8] L. Zhang, VirtualClock: A new traffic control algorithm for packet switching networks, ACM Computer Communications Review 12 (4) (1990) 19-29.

[9] G.X. Geoffery, S.L. Simon, Delay guarantee in virtual clock server, IEEE/ACM Trans. on Networking 3 (6) (1995) 683-689. 
[10] D. Ferrari, D. Verma, A scheme for real-time channel establishment in wide-area networks, IEEE JSAC (1990) 368-379.

[11] D. Verma, H. Zhang, D. Ferrari, Guaranteeing delay jitter bounds in packet switching networks, in: Proc. TRICOMM, April 1991, pp. 3546.

[12] H. Zhang, D. Ferrari, Rate-controlled static-priority queueing, in: Proc. IEEE INFOCOM 1993, San Francisco, California, pp. 227-236.

[13] S.J Golestani, Congestion-free transmission of real-time traffic of real-time traffic in packet networks, IEEE INFOCOM (1990) 527536.

[14] S.J. Golestani, A framing strategy for congestion management, IEEE JSAC 9 (7) (1991) 1064-1077.

[15] S.J. Golestani, Congestion-free communication in high-speed packet networks, IEEE Trans. on Communications 39 (12) (1991) 18021812.
[16] S.J. Golestani, A stop-and-go queueing framework for congestion management, ACM SIGCOMM (1992) 8-18.

[17] B.-J. Tsaur, J.-H. Huang, Continuous framing mechanism for congestion control in broadband networks, Computer Communications 18 (10) (1995) 718-724.

[18] S. Deering, D. Estrin, D. Farinacci, V. Jacobson, C.-G. Liu, L. Wei, An architecture for wide-area multicasting routing, ACM SIGCOMM, 1994 126-135.

[19] A.K.J. Parekh, R.G. Gallager, A generalized processor sharing approach to flow control- the single node case, in: Proc. IEEE INFOCOM, 1992.

[20] A.K.J. Parekh, R.G. Gallager, A generalized processor sharing approach to flow control in integrated services networks: the multiple node case, in: Proc. IEEE INFOCOM, 1993, San Francisco, California, pp. 521-530. 